IMA Journal of Numerical Analysis (2018) Page 1 of 22

doi:10.1093/imanum/drnxxx

\title{
Pointwise-in-Time Error Estimates for an Optimal Control Problem with Subdiffusion Constraint
}

\author{
BANGTI JIN † \\ Department of Computer Science, University College London \\ Gower Street, London WC1E 6BT, UK. \\ BUYANG LI AND ZHI ZHOU $\ddagger$ \\ Department of Applied Mathematics, The Hong Kong Polytechnic University, Hong Kong.
}

[Received on December 22, 2017; revised on June 4, 2018; accepted on ]

\begin{abstract}
In this work, we present numerical analysis for a distributed optimal control problem, with box constraint on the control, governed by a subdiffusion equation which involves a fractional derivative of order $\alpha \in(0,1)$ in time. The fully discrete scheme is obtained by applying the conforming linear Galerkin finite element method in space, L1 scheme/backward Euler convolution quadrature in time, and the control variable by a variational type discretization. With a space mesh size $h$ and time stepsize $\tau$, we establish the following order of convergence for the numerical solutions of the optimal control problem: $O\left(\tau^{\min (1 / 2+\alpha-\varepsilon, 1)}+h^{2}\right)$ in the discrete $L^{2}\left(0, T ; L^{2}(\Omega)\right)$ norm and $O\left(\tau^{\alpha-\varepsilon}+\ell_{h}^{2} h^{2}\right)$ in the discrete $L^{\infty}\left(0, T ; L^{2}(\Omega)\right)$ norm, with any small $\varepsilon>0$ and $\ell_{h}=\ln (2+1 / h)$. The analysis relies essentially on the maximal $L^{p}$-regularity and its discrete analogue for the subdiffusion problem. Numerical experiments are provided to support the theoretical results.
\end{abstract}

Keywords: optimal control, time-fractional diffusion, L1 scheme, convolution quadrature, pointwise-intime error estimate, maximal regularity.

\section{Introduction}

Let $\Omega \subset \mathbb{R}^{d}(d=1,2,3)$ be a convex polyhedral domain with a boundary $\partial \Omega$. Consider the distributed optimal control problem

$$
\min _{q \in U_{\mathrm{ad}}} J(u, q)=\frac{1}{2}\left\|u-u_{d}\right\|_{L^{2}\left(0, T ; L^{2}(\Omega)\right)}^{2}+\frac{\gamma}{2}\|q\|_{L^{2}\left(0, T ; L^{2}(\Omega)\right)}^{2},
$$

subject to the following time-fractional diffusion equation

$$
{ }_{0} \partial_{t}^{\alpha} u-\Delta u=f+q, \quad 0<t \leqslant T, \quad \text { with } u(0)=0,
$$

where $T>0$ is a fixed final time, $\gamma>0$ a fixed penalty parameter, $\Delta: H_{0}^{1}(\Omega) \cap H^{2}(\Omega) \rightarrow L^{2}(\Omega)$ the Dirichlet Laplacian, $f:(0, T) \rightarrow L^{2}(\Omega)$ a given source term, and $u_{d}:(0, T) \rightarrow L^{2}(\Omega)$ the target function. The admissible set $U_{\text {ad }}$ for the control $q$ is defined by

$$
U_{\mathrm{ad}}=\left\{q \in L^{2}\left(0, T ; L^{2}(\Omega)\right): a \leqslant q \leqslant b \text { a.e. in } \Omega \times(0, T)\right\},
$$

with $a, b \in \mathbb{R}$ and $a<b$. The notation ${ }_{0} \partial_{t}^{\alpha} u$ in (1.2) denotes the left-sided Riemann-Liouville fractional derivative in time $t$ of order $\alpha \in(0,1)$, defined by (Kilbas et al., 2006, p. 70)

$$
{ }_{0} \partial_{t}^{\alpha} u(t)=\frac{1}{\Gamma(1-\alpha)} \frac{\mathrm{d}}{\mathrm{d} t} \int_{0}^{t}(t-s)^{-\alpha} u(s) \mathrm{d} s .
$$

\footnotetext{
$\dagger$ Corresponding author. Email: bangti.jin@gmail.com, b.jin@ucl.ac.uk
}

‡Email: bygli@polyu.edu.hk, zhizhou@ polyu.edu.hk

(c) The author 2018. Published by Oxford University Press on behalf of the Institute of Mathematics and its Applications. All rights reserved. 
Since $u(0)=0$, the Riemann-Liouville derivative ${ }_{0} \partial_{t}^{\alpha} u(t)$ is identical to the Caputo derivative (Kilbas et al., 2006, p. 91). Further, when $\alpha=1,{ }_{0} \partial_{t}^{\alpha} u(t)$ coincides with the first-order derivative $u^{\prime}(t)$, and thus the model (1.2) recovers the standard parabolic problem.

The fractional derivative ${ }_{0} \partial_{t}^{\alpha} u$ in the model (1.2) is motivated by a growing list of practical applications related to subdiffusion processes, in which the mean square particle displacement grows sublinearly with time $t$, as opposed to linear growth for normal diffusion. The list includes thermal diffusion in fractal media, protein transport in plasma membrane and column experiments etc (see, e.g., Adams \& Gelhar (1992); Hatano \& Hatano (1998); Nigmatulin (1986)). The numerical analysis of the model (1.2) has received much attention. However, the design and analysis of numerical methods for related optimal control problems only started to attract attention (see, e.g., Antil et al. (2016); Du et al. (2016); Ye \& $\mathrm{Xu}(2013,2015))$. The controllability of (1.2) was discussed in Fujishiro \& Yamamoto (2014) and Lü \& Zuazua (2016). Ye \& Xu $(2013,2015)$ proposed space-time spectral type methods for optimal control problems under a subdiffusion constraint, and derived error estimates by assuming sufficiently smooth state and control variables. Antil et al. (2016) studied an optimal control problem with space- and time-fractional models, and showed the convergence of the discrete approximations via a compactness argument. However, no error estimate for the optimal control was given for the time-fractional case. Zhou \& Gong (2016) proved the well-posedness of problem (1.1)-(1.2) and derived $L^{2}\left(0, T ; L^{2}(\Omega)\right.$ ) error estimates for the spatially semidiscrete finite element method, and described a time discretization method without error estimate. To the best of our knowledge, there is no error estimate for time discretizations of (1.1)-(1.2). It is the main goal of this work to fill this gap.

This work is devoted to the error analysis of both time and space discretizations of (1.1)-(1.2). The model (1.2) is discretized by the continuous piecewise linear Galerkin FEM in space and the L1 approximation (Lin \& Xu (2007)) or backward Euler convolution quadrature (Lubich (1986)) in time, and the control $q$ by a variational type discretization due to Hinze (2005). The analysis relies crucially on $\ell^{p}\left(L^{2}(\Omega)\right)$ error estimates for fully discrete solutions of the direct problem with a nonsmooth source term. Such results are still unavailable in the literature. We derive such estimates in Theorems 2.2 and 2.3, and use them to derive an $O\left(\tau^{\min (1 / 2+\alpha-\varepsilon, 1)}+h^{2}\right)$ error estimate in the discrete $L^{2}\left(0, T ; L^{2}(\Omega)\right)$ norm for the numerical solutions of problem (1.1)-(1.2), where $h$ and $\tau$ denote the mesh size and time stepsize, respectively, and $\varepsilon>0$ is small, cf. Theorems 3.2 and 3.4. The $O\left(\tau^{\min (1 / 2+\alpha-\varepsilon, 1)}\right)$ rate contrasts with the $O(\tau)$ rate for the parabolic counterpart (see, e.g., Meidner \& Vexler (2008); Chrysafinos \& Karatzas (2014); Gong et al. (2014)). The lower rate for $\alpha \leqslant 1 / 2$ is due to the limited smoothing property of problem (1.2), cf. Theorem 2.1. This also constitutes the main technical challenge in the analysis. Based on the error estimate in the discrete $L^{2}\left(0, T ; L^{2}(\Omega)\right)$ norm, we further derive a pointwisein-time error estimate $O\left(\tau^{\alpha-\varepsilon}+\ell_{h}^{2} h^{2}\right)$ (with $\ell_{h}=\log (2+1 / h)$, cf. Theorems 3.3 and 3.5). Our analysis relies essentially on the maximal $L^{p}$-regularity of fractional evolution equations (Bajlekova (2001)) and its discrete analogue (Jin et al. (2018a)). The latter is the fractional extension of the discrete maximal $L^{p}$-regularity theory (Kovács et al. (2016)), which is a mathematical tool for the numerical analysis of nonlinear parabolic equations (Akrivis et al. (2017); Kunstmann et al. (2018)). Numerical experiments in one- and two-dimensional spaces are provided to complement the theoretical analysis.

The rest of the paper is organized as follows. In Section 2, we discuss the solution regularity and numerical approximation for problem (1.2). In Section 3, we prove error bounds on spatially semidiscrete and fully discrete approximations to the optimal control problem (1.1)-(1.2). Finally in Section 4, we provide one- and two-dimensional numerical experiments to support the theoretical results. Throughout, the notation $c$ denotes a generic constant which may differ at each occurrence, but it is always independent of the mesh size $h$ and time stepsize $\tau$.

\section{Regularity theory and numerical approximation of the direct problem}

In this section, we recall preliminaries and present analysis for the direct problem

$$
{ }_{0} \partial_{t}^{\alpha} u-\Delta u=g, \quad 0<t \leqslant T, \quad \text { with } \quad u(0)=0,
$$


and its adjoint problem

$$
{ }_{t} \partial_{T}^{\alpha} z-\Delta z=\eta, \quad 0 \leqslant t<T, \quad \text { with } \quad z(T)=0,
$$

where the fractional derivative ${ }_{t} \partial_{T}^{\alpha} z$ is defined in (2.5) below. The adjoint equation follows from (2.6) below and standard integration by parts formula; see (Zhou \& Gong, 2016, Section 3). In the case $\alpha \in(0,1 / 2]$, the initial / terminal condition should be understood properly: for a rough source term $g$, the temporal trace at $t=0 / t=T$ may not exist and the initial condition should be interpreted in a weak sense (see, e.g., Gorenflo et al. (2015)). We refrain from the case of a nonzero initial condition, and leave it to a future work.

\subsection{Sobolev spaces of functions vanishing at $t=0$}

We shall use extensively Bochner-Sobolev spaces $W^{s, p}\left(0, T ; L^{2}(\Omega)\right)$. For any $s \geqslant 0$ and $1 \leqslant p<\infty$, we denote by $W^{s, p}\left(0, T ; L^{2}(\Omega)\right)$ the space of functions $v:(0, T) \rightarrow L^{2}(\Omega)$, with the norm defined by interpolation. Equivalently, the space is equipped with the quotient norm

$$
\|v\|_{W^{s, p}\left(0, T ; L^{2}(\Omega)\right)}:=\inf _{\widetilde{v}}\|\widetilde{v}\|_{W^{s, p}\left(\mathbb{R} ; L^{2}(\Omega)\right)}
$$

where the infimum is taken over all possible extensions $\widetilde{v}$ that extend $v$ from $(0, T)$ to $\mathbb{R}$. For any $0<s<1$, one can define Sobolev-Slobodeckiř seminorm $|\cdot|_{W^{s, p}\left(0, T ; L^{2}(\Omega)\right)}$ by

$$
|v|_{W^{s, p}\left(0, T ; L^{2}(\Omega)\right)}^{p}:=\int_{0}^{T} \int_{0}^{T} \frac{\|v(t)-v(\xi)\|_{L^{2}(\Omega)}^{p}}{|t-\xi|^{1+p s}} \mathrm{~d} t \mathrm{~d} \xi
$$

and the full norm $\|\cdot\|_{W^{s, p}\left(0, T ; L^{2}(\Omega)\right)}$ by

$$
\|v\|_{W^{s, p}\left(0, T ; L^{2}(\Omega)\right)}^{p}=\|v\|_{L^{p}\left(0, T ; L^{2}(\Omega)\right)}^{p}+|v|_{W^{s, p}\left(0, T ; L^{2}(\Omega)\right)}^{p} .
$$

For $s>1$, one can define similar seminorms and norms. Let

$$
C_{L}^{\infty}\left(0, T ; L^{2}(\Omega)\right):=\left\{v=\left.w\right|_{(0, T)}: w \in C^{\infty}\left(\mathbb{R} ; L^{2}(\Omega)\right): \operatorname{supp}(w) \subset[0, \infty)\right\},
$$

and denote by $W_{L}^{s, p}\left(0, T ; L^{2}(\Omega)\right)$ the closure of $C_{L}^{\infty}\left(0, T ; L^{2}(\Omega)\right)$ in $W^{s, p}\left(0, T ; L^{2}(\Omega)\right)$, and by $W_{R}^{s, p}\left(0, T ; L^{2}(\Omega)\right)$ the closure of $C_{R}^{\infty}\left(0, T ; L^{2}(\Omega)\right)$ in $W^{s, p}\left(0, T ; L^{2}(\Omega)\right)$, with

$$
C_{R}^{\infty}\left(0, T ; L^{2}(\Omega)\right):=\left\{v=\left.w\right|_{(0, T)}: w \in C^{\infty}\left(\mathbb{R} ; L^{2}(\Omega)\right): \operatorname{supp}(w) \subset(-\infty, T]\right\} .
$$

By Sobolev embedding, for $v \in W_{L}^{s, p}\left(0, T ; L^{2}(\Omega)\right)$, there holds $v^{(j)}(0)=0$ for $j=0, \ldots,[s]-1$ (with $[s]$ being the integral part of $s>0)$, and also $v^{(j)}=0$ if $(s-[s]) p>1$. For $v \in W_{L}^{s, p}\left(0, T ; L^{2}(\Omega)\right)$, the zero extension of $v$ to the left belongs to $W^{s, p}\left(-\infty, T ; L^{2}(\Omega)\right)$, and $W_{L}^{s, p}\left(0, T ; L^{2}(\Omega)\right)=W^{s, p}\left(0, T ; L^{2}(\Omega)\right)$, if $s<1 / p$. We abbreviate $W_{L}^{s, 2}\left(0, T ; L^{2}(\Omega)\right)$ as $H_{L}^{s}\left(0, T ; L^{2}(\Omega)\right)$, and likewise $H_{R}^{s}\left(0, T ; L^{2}(\Omega)\right)$ for $W_{R}^{s, 2}\left(0, T ; L^{2}(\Omega)\right)$.

Similar to the left-sided fractional derivative ${ }_{0} \partial_{t}^{\alpha} u$ in (1.3), the right-sided Riemann-Liouville fractional derivative ${ }_{t} \partial_{T}^{\alpha} v(t)$ in (2.2) is defined by

$$
{ }_{t} \partial_{T}^{\alpha} v(t):=-\frac{1}{\Gamma(1-\alpha)} \frac{\mathrm{d}}{\mathrm{d} t} \int_{t}^{T}(s-t)^{-\alpha} v(s) \mathrm{d} s .
$$

Let any $p \in(1, \infty)$ and $p^{\prime} \in(1, \infty)$ be conjugate to each other, i.e., $1 / p+1 / p^{\prime}=1$. Since for $u \in$ $W_{L}^{\alpha, p}\left(0, T ; L^{2}(\Omega)\right), v \in W_{R}^{\alpha, p^{\prime}}\left(0, T ; L^{2}(\Omega)\right)$, we have ${ }_{0} \partial_{t}^{\alpha} u \in L^{p}\left(0, T ; L^{2}(\Omega)\right),{ }_{t} \partial_{T}^{\alpha} v \in L^{p^{\prime}}\left(0, T ; L^{2}(\Omega)\right)$. Thus, there holds (Kilbas et al., 2006, p. 76, Lemma 2.7):

$$
\int_{0}^{T}\left({ }_{0} \partial_{t}^{\alpha} u(t)\right) v(t) \mathrm{d} t=\int_{0}^{T} u(t)\left({ }_{t} \partial_{T}^{\alpha} v(t)\right) \mathrm{d} t, \forall u \in W_{L}^{\alpha, p}\left(0, T ; L^{2}(\Omega)\right), v \in W_{R}^{\alpha, p^{\prime}}\left(0, T ; L^{2}(\Omega)\right) .
$$




\subsection{Regularity of the direct problem}

The next maximal $L^{p}$-regularity holds (cf. Bajlekova (2001)), and an analogous result holds for (2.2).

LEMMA 2.1 If $u_{0}=0$ and $g \in L^{p}\left(0, T ; L^{2}(\Omega)\right)$ with $1<p<\infty$, then problem (2.1) has a unique solution $u \in L^{p}\left(0, T ; H_{0}^{1}(\Omega) \cap H^{2}(\Omega)\right)$ such that ${ }_{0} \partial_{t}^{\alpha} u \in L^{p}\left(0, T ; L^{2}(\Omega)\right)$ and

$$
\|u\|_{L^{p}\left(0, T ; H^{2}(\Omega)\right)}+\left\|_{0} \partial_{t}^{\alpha} u\right\|_{L^{p}\left(0, T ; L^{2}(\Omega)\right)} \leqslant c\|g\|_{L^{p}\left(0, T ; L^{2}(\Omega)\right)},
$$

where the constant $c$ is independent of $g$ and $T$.

Now we give a regularity result. Throughout, the notation $\Delta$ denotes the Dirichlet Laplacian, with its domain $D(\Delta)=H_{0}^{1}(\Omega) \cap H^{2}(\Omega)$.

THEOREM 2.1 For $g \in W^{s, p}\left(0, T ; L^{2}(\Omega)\right), s \in[0,1 / p)$ and $p \in(1, \infty)$, problem (2.1) has a unique solution $u \in W^{\alpha+s, p}\left(0, T ; L^{2}(\Omega)\right) \cap W^{s, p}(0, T ; D(\Delta))$, which satisfies

$$
\|u\|_{W^{\alpha+s, p}\left(0, T ; L^{2}(\Omega)\right)}+\|u\|_{W^{s, p}\left(0, T ; H^{2}(\Omega)\right)} \leqslant c\|g\|_{W^{s, p}\left(0, T ; L^{2}(\Omega)\right)} .
$$

Similarly, for $\eta \in W^{s, p}\left(0, T ; L^{2}(\Omega)\right), s \in[0,1 / p)$ and $p \in(1, \infty)$, problem (2.2) has a unique solution $z \in W^{\alpha+s, p}\left(0, T ; L^{2}(\Omega)\right) \cap W^{s, p}(0, T ; D(\Delta))$, which satisfies

$$
\|z\|_{W^{\alpha+s, p}\left(0, T ; L^{2}(\Omega)\right)}+\|z\|_{W^{s, p}\left(0, T ; H^{2}(\Omega)\right)} \leqslant c\|\eta\|_{W^{s, p}\left(0, T ; L^{2}(\Omega)\right)} .
$$

Proof. For $g \in W^{s, p}\left(0, T ; L^{2}(\Omega)\right), s \in[0,1 / p)$ and $p \in(1, \infty)$, extending $g$ to be zero on $\Omega \times[(-\infty, 0) \cup$ $(T, \infty)]$ yields $g \in W^{s, p}\left(\mathbb{R} ; L^{2}(\Omega)\right)$ and

$$
\|g\|_{W^{s, p}\left(\mathbb{R} ; L^{2}(\Omega)\right)} \leqslant c\|g\|_{W^{s, p}\left(0, T ; L^{2}(\Omega)\right)} .
$$

Further, we have the identity ${ }_{0} \partial_{t}^{\alpha} g(t)={ }_{-\infty} \partial_{t}^{\alpha} g(t)$ for $t \in[0, T]$, and $\widehat{{ }_{-\infty} \partial_{t}^{\alpha} g}=(i \xi)^{\alpha} \widehat{g}(\xi)$ (Kilbas et al., 2006 , p. 90), where denotes taking Fourier transform in $t$, and $\widehat{g}$ the Fourier transform of $g$. Then, with ${ }^{\vee}$ being the inverse Fourier transform in $\xi, u=\left[\left((i \xi)^{\alpha}-\Delta\right)^{-1} \widehat{g}(\xi)\right]^{\vee}$ is a solution of (2.1) and

$$
\left(1+|\xi|^{2}\right)^{\frac{\alpha+s}{2}} \widehat{u}(\xi)=\left(1+|\xi|^{2}\right)^{\frac{\alpha}{2}}\left((i \xi)^{\alpha}-\Delta\right)^{-1}\left(1+|\xi|^{2}\right)^{\frac{s}{2}} \widehat{g}(\xi) .
$$

The self-adjoint operator $\Delta: D(\Delta) \rightarrow L^{2}(\Omega)$ is invertible from $L^{2}(\Omega)$ to $D(\Delta)$, and generates a bounded analytic semigroup (Arendt et al., 2011, Example 3.7.5). Thus the operator

$$
\left(1+|\xi|^{2}\right)^{\frac{\alpha}{2}}\left((i \xi)^{\alpha}-\Delta\right)^{-1}
$$

is bounded from $L^{2}(\Omega)$ to $D(\Delta)$ in a small neighborhood $\mathscr{N}$ of $\xi=0$. Further, in $\mathscr{N}$, the operator

$$
\begin{aligned}
\xi \frac{\mathrm{d}}{\mathrm{d} \xi}\left(1+|\xi|^{2}\right)^{\frac{\alpha}{2}}\left((i \xi)^{\alpha}-\Delta\right)^{-1}= & \frac{\alpha|\xi|^{2}}{1+|\xi|^{2}}\left(1+|\xi|^{2}\right)^{\frac{\alpha}{2}}\left((i \xi)^{\alpha}-\Delta\right)^{-1} \\
& +\alpha\left(1+|\xi|^{2}\right)^{\frac{\alpha}{2}}\left((i \xi)^{\alpha}-\Delta\right)^{-1}(i \xi)^{\alpha}\left((i \xi)^{\alpha}-\Delta\right)^{-1}
\end{aligned}
$$

is also bounded. If $\xi$ is away from 0 , then

$$
\left(1+|\xi|^{2}\right)^{\frac{\alpha}{2}}\left((i \xi)^{\alpha}-\Delta\right)^{-1}=(i \xi)^{-\alpha}\left(1+|\xi|^{2}\right)^{\frac{\alpha}{2}}(i \xi)^{\alpha}\left((i \xi)^{\alpha}-\Delta\right)^{-1}
$$

Now we slightly abuse the notation $\|\cdot\|$ for the operator norm on $L^{2}(\Omega)$. Recall also the resolvent estimate $\left\|(z-\Delta)^{-1}\right\| \leqslant c|z|^{-1}$ for any $z \in \Sigma_{\theta}:=\{z \in \mathbb{C}: z \neq 0,|\arg (z)| \leqslant \theta\}$, for all $\theta \in(\pi / 2, \pi)$ (Arendt et al., 2011, Example 3.7.5 and Theorem 3.7.11). Then, the following inequalities

$$
\left|(i \xi)^{-\alpha}\left(1+|\xi|^{2}\right)^{\frac{\alpha}{2}}\right| \leqslant c \text { and }\left\|(i \xi)^{\alpha}\left((i \xi)^{\alpha}-\Delta\right)^{-1}\right\| \leqslant c
$$


imply the boundedness of (2.8) and (2.9).

Since boundedness of operators is equivalent to $R$-boundedness of operators in $L^{2}(\Omega)$ (see Kunstmann \& Weis (2004) for the concept of $R$-boundedness), the boundedness of (2.8) and (2.9) implies that (2.8) is an operator-valued Fourier multiplier (Weis, 2001, Theorem 3.4), and thus

$$
\begin{aligned}
\|u\|_{W^{\alpha+s, p}\left(\mathbb{R} ; L^{2}(\Omega)\right)} & \leqslant\left\|\left[\left(1+|\xi|^{2}\right)^{\frac{\alpha+s}{2}} \widehat{u}(\xi)\right]^{\vee}\right\|_{L^{p}\left(\mathbb{R} ; L^{2}(\Omega)\right)} \\
& =\left\|\left[\left(1+|\xi|^{2}\right)^{\frac{\alpha}{2}}\left((i \xi)^{\alpha}-\Delta\right)^{-1}\left(1+|\xi|^{2}\right)^{\frac{s}{2}} \widehat{g}(\xi)\right]^{\vee}\right\|_{L^{p}\left(\mathbb{R} ; L^{2}(\Omega)\right)} \\
& \leqslant c\left\|\left[\left(1+|\xi|^{2}\right)^{\frac{s}{2}} \widehat{g}(\xi)\right]^{\vee}\right\|_{L^{p}\left(\mathbb{R} ; L^{2}(\Omega)\right)} \leqslant c\|g\|_{W^{s, p}\left(\mathbb{R} ; L^{2}(\Omega)\right)} .
\end{aligned}
$$

This and (2.7) imply the desired bound on $\|u\|_{W^{\alpha+s, p\left(0, T ; L^{2}(\Omega)\right)}}$. The estimate

$$
\|u\|_{W^{s, p}\left(0, T ; H^{2}(\Omega)\right)} \leqslant c\|g\|_{W^{s, p}\left(0, T ; L^{2}(\Omega)\right)}
$$

follows similarly by replacing $\left(1+|\xi|^{2}\right)^{\frac{\alpha}{2}}\left((i \xi)^{\alpha}-\Delta\right)^{-1}$ with $\Delta\left((i \xi)^{\alpha}-\Delta\right)^{-1}$ in the proof.

REMARK 2.1 Below we only use the cases " $p=2, s=\min (1 / 2-\varepsilon, \alpha-\varepsilon)$ " and " $p>\max (1 / \alpha, 1 /(1-$ $\alpha)), s=1 / p-\varepsilon$ " of Theorem 2.1. Both cases satisfy the conditions of Theorem 2.1. A similar assertion holds for the more general case $g \in W_{L}^{s, p}\left(0, T ; L^{2}(\Omega)\right), s>0$ and $1<p<\infty$ :

$$
\|u\|_{W^{\alpha+s, p}\left(0, T ; L^{2}(\Omega)\right)}+\|u\|_{W^{s, p}\left(0, T ; H^{2}(\Omega)\right)} \leqslant c\|g\|_{W_{L}^{s, p}\left(0, T ; L^{2}(\Omega)\right)} .
$$

In fact, for $g \in W_{L}^{s, p}\left(0, T ; L^{2}(\Omega)\right)$, the zero extension of $g$ to $t \leqslant 0$ belongs to $W^{s, p}\left(-\infty, T ; L^{2}(\Omega)\right)$, which can further be boundedly extended to a function in $W^{s, p}\left(\mathbb{R} ; L^{2}(\Omega)\right)$. Then the argument in Theorem 2.1 gives the desired assertion. This also indicates a certain compatibility condition for regularity pickup.

\subsection{Numerical scheme for problem (2.1)}

Now we describe numerical treatment of the forward problem (2.1), which forms the basis for the fully discrete scheme of the control problem (1.1)-(1.2) in Section 3. We denote by $\mathscr{T}_{h}$ a shape-regular and quasi-uniform triangulation of the domain $\Omega$ into $d$-dimensional simplexes, and let

$$
X_{h}=\left\{v_{h} \in H_{0}^{1}(\Omega):\left.v_{h}\right|_{K} \text { is a linear function, } \forall K \in \mathscr{T}_{h}\right\}
$$

be the finite element space consisting of continuous piecewise linear functions. The $L^{2}(\Omega)$-orthogonal projection $P_{h}: L^{2}(\Omega) \rightarrow X_{h}$ is defined by $\left(P_{h} \varphi, \chi_{h}\right)=\left(\varphi, \chi_{h}\right)$, for all $\varphi \in L^{2}(\Omega), \chi_{h} \in X_{h}$, where $(\cdot, \cdot)$ denotes the $L^{2}(\Omega)$ inner product. Then the spatially semidiscrete Galerkin FEM for problem (2.1) is to find $u_{h}(t) \in X_{h}$ such that $u_{h}(0)=0$ and

$$
\left({ }_{0} \partial_{t}^{\alpha} u_{h}(t), \chi_{h}\right)+\left(\nabla u_{h}(t), \nabla \chi_{h}\right)=\left(g(t), \chi_{h}\right), \quad \forall \chi_{h} \in X_{h}, \forall t \in(0, T]
$$

By introducing the discrete Laplacian $\Delta_{h}: X_{h} \rightarrow X_{h}$, defined by $-\left(\Delta_{h} \varphi_{h}, \chi_{h}\right)=\left(\nabla \varphi_{h}, \nabla \chi_{h}\right)$, for all $\varphi_{h}, \chi_{h} \in X_{h}$, problem (2.10) can be written as

$$
{ }_{0} \partial_{t}^{\alpha} u_{h}(t)-\Delta_{h} u_{h}(t)=P_{h} g(t), \quad \forall t \in(0, T], \quad \text { with } u_{h}(0)=0
$$

Similar to Theorem 2.1, for $s<1 / p$ there holds

$$
\left\|u_{h}\right\|_{W^{\alpha+s, p}\left(0, T ; L^{2}(\Omega)\right)}+\left\|\Delta_{h} u_{h}\right\|_{W^{s, p}\left(0, T ; L^{2}(\Omega)\right)} \leqslant c\|g\|_{W^{s, p}\left(0, T ; L^{2}(\Omega)\right)},
$$

where the constant $c$ is independent of $h$ (following the proof of Theorem 2.1). Lemma 2.1 and Remark 2.1 remain valid for the semidiscrete solution $u_{h}$, e.g.,

$$
\left\|\Delta_{h} u_{h}\right\|_{L^{p}\left(0, T ; L^{2}(\Omega)\right)}+\left\|_{0} \partial_{t}^{\alpha} u_{h}\right\|_{L^{p}\left(0, T ; L^{2}(\Omega)\right)} \leqslant c\|g\|_{L^{p}\left(0, T ; L^{2}(\Omega)\right)} .
$$


These assertions will be used extensively below without explicitly referencing.

To discretize (2.11) in time, we uniformly partition $[0, T]$ with grid points $t_{n}=n \tau, n,=0,1,2, \ldots, N$ and a time stepsize $\tau=T / N \leqslant 1$, and approximate ${ }_{0} \partial_{t}^{\alpha} \varphi\left(t_{n}\right)$ by (with $\varphi^{j}=\varphi\left(t_{j}\right)$ ):

$$
{ }_{0} \bar{\partial}_{\tau}^{\alpha} \varphi^{n}=\tau^{-\alpha} \sum_{j=0}^{n} \beta_{n-j} \varphi^{j}
$$

where $\beta_{j}$ are suitable weights. We consider two time stepping schemes: L1 scheme (Lin \& Xu (2007)) and backward Euler convolution quadrature (BE-CQ) (Lubich (1986)), for which $\beta_{j}$ are respectively given by (with $c_{\alpha}=1 / \Gamma(2-\alpha)$ )

L1 scheme: $\quad \beta_{0}=c_{\alpha}, \quad$ and $\beta_{j}=c_{\alpha}\left((j+1)^{1-\alpha}-2 j^{1-\alpha}+(j-1)^{1-\alpha}\right), j=1,2, \ldots, N$,

BE-CQ: $\quad \beta_{0}=1, \quad$ and $\beta_{j}=-\beta_{j-1}(\alpha-j+1) / j, j=1,2, \ldots, N$.

Both schemes extend the classical backward Euler scheme to the fractional case. Then we discretize problem (2.1) by: with $g_{h}^{n}=P_{h} g\left(t_{n}\right)$, find $U_{h}^{n} \in X_{h}$ such that

$$
{ }_{0} \bar{\partial}_{\tau}^{\alpha} U_{h}^{n}-\Delta_{h} U_{h}^{n}=g_{h}^{n}, \quad n=1,2, \ldots, N, \quad \text { with } U_{h}^{0}=0 .
$$

By (Jin et al., 2018b, Section 5) and (Jin et al., 2016, Theorem 3.6), we have the following error bound.

LEMMA 2.2 For $g \in W^{1, p}\left(0, T ; L^{2}(\Omega)\right), 1 \leqslant p \leqslant \infty$, let $u_{h}$ and $U_{h}^{n}$ be the semidiscrete solution and fully discrete solution, respectively, in (2.11) and (2.15). Then there holds

$$
\left\|U_{h}^{n}-u_{h}\left(t_{n}\right)\right\|_{L^{2}(\Omega)} \leqslant c \tau t_{n}^{\alpha-1}\|g(0)\|_{L^{2}(\Omega)}+c \tau \int_{0}^{t_{n}}\left(t_{n+1}-s\right)^{\alpha-1}\left\|g^{\prime}(s)\right\|_{L^{2}(\Omega)} \mathrm{d} s .
$$

REMARK 2.2 Lemma 2.2 slightly refines the estimates in Jin et al. (2018b, 2016), but can be proved in the same way using the following estimates in the proof of (Jin et al., 2018b, Section 5):

$$
t_{n}^{\alpha-1} \leqslant c(t+\tau)^{\alpha-1} \text { and } \int_{t}^{t_{n}} s^{\alpha-1} \mathrm{~d} s \leqslant c \tau(t+\tau)^{\alpha-1}, \text { for } t \in\left[t_{n-1}, t_{n}\right] \text { and } n=1, \ldots, N .
$$

For any Banach space $X$, we define

$$
\left\|\left(U_{h}^{n}\right)_{n=0}^{N}\right\|_{\ell^{p}(X)}:= \begin{cases}\left(\sum_{n=0}^{N} \tau\left\|U_{h}^{n}\right\|_{X}^{p}\right)^{1 / p} & \text { if } 1 \leqslant p<\infty, \\ \max _{0 \leqslant n \leqslant N}\left\|U_{h}^{n}\right\|_{X} & \text { if } p=\infty .\end{cases}
$$

Then the maximal $\ell^{p}$-regularity estimate holds for (2.15) (Jin et al., 2018a, Theorems 5 and 7).

LEMma 2.3 The solutions $\left(U_{h}^{n}\right)_{n=1}^{N}$ of (2.15) satisfy

$$
\left\|\left({ }_{0} \bar{\partial}_{\tau}^{\alpha} U_{h}^{n}\right)_{n=1}^{N}\right\|_{\ell p\left(L^{2}(\Omega)\right)}+\left\|\left(\Delta_{h} U_{h}^{n}\right)_{n=1}^{N}\right\|_{\ell p\left(L^{2}(\Omega)\right)} \leqslant c_{p}\left\|\left(g_{h}^{n}\right)_{n=1}^{N}\right\|_{\ell p\left(L^{2}(\Omega)\right)}, \quad \forall 1<p<\infty .
$$

\subsection{Error estimates}

Now we present $\ell^{p}\left(L^{2}(\Omega)\right)$ error estimates for $g \in W^{s, p}\left(0, T ; L^{2}(\Omega)\right), 0 \leqslant s \leqslant 1,1 \leqslant p \leqslant \infty$. Error analysis for such $g$ is still unavailable. We need an interpolation error estimate. This result seems standard, but we are unable to find a proof, and thus include a proof in Appendix A.

Lemma 2.4 For $v \in W^{s, p}\left(0, T ; L^{2}(\Omega)\right), 1<p<\infty$ and $s \in(1 / p, 1]$, let $\bar{v}^{n}=\tau^{-1} \int_{t_{n-1}}^{t_{n}} v(t) \mathrm{d} t$. Then there holds

$$
\left\|\left(v\left(t_{n}\right)-\bar{v}^{n}\right)_{n=1}^{N}\right\|_{\ell^{p}\left(L^{2}(\Omega)\right)} \leqslant c \tau^{s}\|v\|_{W^{s, p}\left(0, T ; L^{2}(\Omega)\right)} .
$$


Our first result is an error estimate for $g \in W_{L}^{s, p}\left(0, T ; L^{2}(\Omega)\right)$ (i.e., compatible source). Since $g$ may not be smooth enough in time for pointwise evaluation, we define the averages $\bar{g}_{h}^{n}=\tau^{-1} \int_{t_{n-1}}^{t_{n}} P_{h} g(s) \mathrm{d} s$, and consider a variant of the scheme (2.15) for problem (2.1): find $\bar{U}_{h}^{n} \in X_{h}$ such that

$$
{ }_{0} \bar{\partial}_{\tau}^{\alpha} \bar{U}_{h}^{n}-\Delta_{h} \bar{U}_{h}^{n}=\bar{g}_{h}^{n}, \quad n=1, \ldots, N, \quad \text { with } \bar{U}_{h}^{0}=0
$$

THEOREM 2.2 For $g \in W_{L}^{s, p}\left(0, T ; L^{2}(\Omega)\right), 1<p<\infty$ and $s \in[0,1]$, let $u_{h}$ and $\bar{U}_{h}^{n}$ be the solutions of problems (2.11) and (2.16), respectively, and $\bar{u}_{h}^{n}:=\tau^{-1} \int_{t_{n-1}}^{t_{n}} u_{h}(s) \mathrm{d} s$. Then there holds

$$
\left\|\left(\bar{U}_{h}^{n}-\bar{u}_{h}^{n}\right)_{n=1}^{N}\right\|_{\ell p\left(L^{2}(\Omega)\right)} \leqslant c \tau^{s}\|g\|_{W_{L}^{s, p}\left(0, T ; L^{2}(\Omega)\right)} .
$$

Proof. By Hölder's inequality and (2.12) (with $s=0$ ), we have

$$
\begin{aligned}
\tau \sum_{n=1}^{N}\left\|\bar{u}_{h}^{n}\right\|_{L^{2}(\Omega)}^{p} & =\tau \sum_{n=1}^{N}\left\|\tau^{-1} \int_{t_{n-1}}^{t_{n}} u_{h}(s) \mathrm{d} s\right\|_{L^{2}(\Omega)}^{p} \leqslant \tau^{1-p} \sum_{n=1}^{N}\left(\int_{t_{n-1}}^{t_{n}}\left\|u_{h}(s)\right\|_{L^{2}(\Omega)} \mathrm{d} s\right)^{p} \\
& \leqslant \int_{0}^{T}\left\|u_{h}(s)\right\|_{L^{2}(\Omega)}^{p} \mathrm{~d} s \leqslant c\|g\|_{L^{p}\left(0, T ; L^{2}(\Omega)\right)}^{p}
\end{aligned}
$$

Similarly, by applying Lemma 2.3 to (2.16) and the $L^{2}(\Omega)$ stability of $P_{h}$, we have

$$
\left\|\left(\bar{U}_{h}^{n}\right)_{n=1}^{N}\right\|_{\ell^{p}\left(L^{2}(\Omega)\right)} \leqslant c\left\|\left(\bar{g}_{h}^{n}\right)_{n=1}^{N}\right\|_{\ell^{p}\left(L^{2}(\Omega)\right)} \leqslant c\|g\|_{L^{p}\left(0, T ; L^{2}(\Omega)\right)}
$$

This and the triangle inequality show the assertion for $s=0$.

Next we consider $g \in W_{L}^{1, p}\left(0, T ; L^{2}(\Omega)\right)$, and resort to (2.15). Since $g(0)=0$, by Lemma 2.2,

$$
\left\|U_{h}^{n}-u_{h}\left(t_{n}\right)\right\|_{L^{2}(\Omega)} \leqslant c \tau \int_{0}^{t_{n}}\left(t_{n}+\tau-s\right)^{\alpha-1}\left\|g^{\prime}(s)\right\|_{L^{2}(\Omega)} \mathrm{d} s
$$

This directly implies

$$
\left\|\left(U_{h}^{n}-u_{h}\left(t_{n}\right)\right)_{n=1}^{N}\right\|_{\ell^{\infty}\left(L^{2}(\Omega)\right)} \leqslant c \tau\|g\|_{W_{L}^{1, \infty}\left(0, T ; L^{2}(\Omega)\right)} .
$$

Further, let $\psi(s)=\tau \sum_{n=1}^{N}\left(t_{n}+\tau-s\right)^{\alpha-1} \chi_{\left[0, t_{n}\right]}$, where $\chi_{S}$ denotes the characteristic function of a set $S$. Then clearly, we have

$$
\sup _{s \in[0, T]} \psi(s)=\psi(T)=\tau \sum_{n=1}^{N}(n \tau)^{\alpha-1} \leqslant \int_{0}^{T} s^{\alpha-1} \mathrm{~d} s=\alpha^{-1} T^{\alpha} \leqslant c_{T} .
$$

Therefore,

$$
\begin{aligned}
\left\|\left(U_{h}^{n}-u_{h}\left(t_{n}\right)\right)_{n=1}^{N}\right\|_{\ell^{1}\left(L^{2}(\Omega)\right)} & \leqslant c \tau^{2} \sum_{n=1}^{N} \int_{0}^{t_{n}}\left(t_{n}+\tau-s\right)^{\alpha-1}\left\|g^{\prime}(s)\right\|_{L^{2}(\Omega)} \mathrm{d} s \\
& =c \tau \int_{0}^{T} \psi(s)\left\|g^{\prime}(s)\right\|_{L^{2}(\Omega)} \mathrm{d} s \leqslant c_{T} \tau\|g\|_{W^{1,1}\left(0, T ; L^{2}(\Omega)\right)} .
\end{aligned}
$$

Then (2.17), (2.18) and Riesz-Thorin interpolation theorem (Bergh \& Lofstrom, 2012, Theorem 1.1.1), yield for any $1<p<\infty$

$$
\left\|\left(U_{h}^{n}-u_{h}\left(t_{n}\right)\right)_{n=1}^{N}\right\|_{\ell p}\left(L^{2}(\Omega)\right) \leqslant c \tau\|g\|_{W_{L}^{1, p}\left(0, T ; L^{2}(\Omega)\right)} .
$$

Since $U_{h}^{n}-\bar{U}_{h}^{n}$ satisfies the discrete scheme, cf. (2.15) and (2.16), Lemmas 2.3 and 2.4 imply

$$
\left\|\left(U_{h}^{n}-\bar{U}_{h}^{n}\right)_{n=1}^{N}\right\|_{\ell^{p}\left(L^{2}(\Omega)\right)} \leqslant c\left\|\left(\bar{g}_{h}^{n}-P_{h} g\left(t_{n}\right)\right)_{n=1}^{N}\right\|_{\ell^{p}\left(L^{2}(\Omega)\right)} \leqslant c \tau\|g\|_{W^{1, p}\left(0, T ; L^{2}(\Omega)\right)} .
$$


Further, by Lemma 2.4 and Remark 2.1, we have

$$
\left\|\left(u_{h}\left(t_{n}\right)-\bar{u}_{h}^{n}\right)_{n=1}^{N}\right\|_{\ell p\left(L^{2}(\Omega)\right)} \leqslant c \tau\left\|u_{h}\right\|_{W^{1, p}\left(0, T ; L^{2}(\Omega)\right)} \leqslant c \tau\|g\|_{W_{L}^{1, p}\left(0, T ; L^{2}(\Omega)\right)} .
$$

The last three estimates show the assertion for $s=1$. The case $0<s<1$ follows by interpolation.

In Theorem 2.2, we compare the numerical solution $\bar{U}_{h}^{n}$ to (2.16) with the time-averaged solution $\bar{u}_{h}^{n}$, instead of $u_{h}\left(t_{n}\right)$. This is due to possible insufficient temporal regularity of $u_{h}$ : it is unclear how to define $u_{h}\left(t_{n}\right)$ for $t_{n} \in(0, T]$ for $g \in W_{L}^{s, p}\left(0, T ; L^{2}(\Omega)\right)$ with $s+\alpha<1 / p$. For $s \in(1 / p, 1], W_{L}^{s, p}\left(0, T ; L^{2}(\Omega)\right) \hookrightarrow$ $C\left([0, T] ; L^{2}(\Omega)\right)$ and so Theorem 2.2 requires the condition $g(0)=0$. Such a compatibility condition at $t=0$ is not necessarily satisfied by problem (1.1)-(1.2). Hence, we state an error estimate below for a smooth but incompatible source $g \in W^{s, p}\left(0, T ; L^{2}(\Omega)\right)$.

TheOREM 2.3 For $g \in W^{s, p}\left(0, T ; L^{2}(\Omega)\right)$ with $p \in(1, \infty)$ and $s \in(1 / p, 1)$, let $u_{h}$ and $U_{h}^{n}$ be the solutions of problems (2.11) and (2.15), respectively. Then there holds

$$
\left\|\left(U_{h}^{n}-u_{h}\left(t_{n}\right)\right)_{n=1}^{N}\right\|_{\ell p\left(L^{2}(\Omega)\right)} \leqslant c \tau^{\min (1 / p+\alpha, s)}\|g\|_{W^{s, p}\left(0, T ; L^{2}(\Omega)\right)} .
$$

Moreover, if $p>1 / \alpha$ is so large that $\alpha \in\left(0,1 / p^{\prime}\right)$, then

$$
\left\|\left(U_{h}^{n}-u_{h}\left(t_{n}\right)\right)_{n=1}^{N}\right\|_{\ell^{\infty}\left(L^{2}(\Omega)\right)} \leqslant c \tau^{\alpha}\|g\|_{W^{1 / p+\alpha, p}\left(0, T ; L^{2}(\Omega)\right)} .
$$

Proof. For $g(x, t) \equiv g(x)$, which belongs to $W^{1, p}\left(0, T ; L^{2}(\Omega)\right)$, by Lemma 2.2 we have

$$
\begin{aligned}
\left\|\left(U_{h}^{n}-u_{h}\left(t_{n}\right)\right)_{n=1}^{N}\right\|_{\ell p\left(L^{2}(\Omega)\right)}^{p} & =\tau \sum_{n=1}^{N}\left\|U_{h}^{n}-u_{h}\left(t_{n}\right)\right\|_{L^{2}(\Omega)}^{p} \leqslant c \tau^{p+1}\|g\|_{L^{2}(\Omega)}^{p} \sum_{n=1}^{N} t_{n}^{p(\alpha-1)} \\
& \leqslant c \tau^{p \alpha+1}\|g\|_{L^{2}(\Omega)}^{p}+c \tau^{p}\|g\|_{L^{2}(\Omega)}^{p} \int_{\tau}^{T} t^{p(\alpha-1)} \mathrm{d} t .
\end{aligned}
$$

Now for $s<1$, there holds

$$
\tau^{p} \int_{\tau}^{T} t^{p(\alpha-1)} \mathrm{d} t \leqslant\left\{\begin{array}{ll}
c \tau^{p \alpha+1} & \text { if } \alpha \in\left(0,1 / p^{\prime}\right) \\
c \tau^{p} & \text { if } \alpha \in\left(1 / p^{\prime}, 1\right) \\
c \tau^{p}(1+\ln (T / \tau)) & \text { if } \alpha=1 / p^{\prime}
\end{array} \leqslant \begin{cases}c \tau^{p(1 / p+\alpha)} & \text { if } \alpha \in\left(0,1 / p^{\prime}\right) \\
c \tau^{p s} & \text { if } \alpha \in\left(1 / p^{\prime}, 1\right) \\
c \tau^{p s} & \text { if } \alpha=1 / p^{\prime}\end{cases}\right.
$$

which together with the preceding estimate implies

$$
\left\|\left(U_{h}^{n}-u_{h}\left(t_{n}\right)\right)_{n=1}^{N}\right\|_{\ell p\left(L^{2}(\Omega)\right)} \leqslant c \tau^{\min (1 / p+\alpha, s)}\|g\|_{W^{s, p}\left(0, T ; L^{2}(\Omega)\right)} .
$$

For $g \in W^{s, p}\left(0, T ; L^{2}(\Omega)\right)$, by Sobolev embedding, $g(0)$ exists, and in the splitting $g(t)=g(0)+(g(t)-$ $g(0))$, there holds $\|g-g(0)\|_{W_{L}^{s, p}\left(0, T ; L^{2}(\Omega)\right)} \leqslant c\|g\|_{W^{s, p}\left(0, T ; L^{2}(\Omega)\right)}$. Let $v_{h}$ be the semidiscrete solution for the source $g(t)-g(0)$, and $\bar{v}_{h}^{h}=\int_{t_{n-1}}^{t_{n}} v_{h}(t) \mathrm{d} t$. Since $g(t)-g(0) \in W_{L}^{s, p}\left(0, T ; L^{2}(\Omega)\right)$, by Theorem 2.2, the corresponding fully discrete solution $\bar{V}_{h}^{n}$ by (2.16) satisfies

$$
\left\|\left(\bar{V}_{h}^{n}-\bar{v}_{h}^{n}\right)_{n=1}^{N}\right\|_{\ell p\left(L^{2}(\Omega)\right)} \leqslant c \tau^{s}\|g-g(0)\|_{W_{L}^{s, p}\left(0, T ; L^{2}(\Omega)\right)} .
$$

Further, by Lemma 2.4 and (2.12), we have



Similarly, for the fully discrete solution $V_{h}^{n}$ corresponding to the source $g(t)-g(0)$ by (2.15), from Lemmas 2.3 and 2.4 , we deduce

$$
\begin{aligned}
\left\|\left(V_{h}^{n}-\bar{V}_{h}^{n}\right)_{n=1}^{N}\right\|_{\ell^{p}\left(L^{2}(\Omega)\right)} & \leqslant c\left\|\left(P_{h} g\left(t_{n}\right)-\bar{g}_{h}^{n}\right)_{n=1}^{N}\right\|_{\ell^{p}\left(L^{2}(\Omega)\right)} \\
& \leqslant c \tau^{s}\|g\|_{W^{s, p}\left(0, T ; L^{2}(\Omega)\right)} .
\end{aligned}
$$


These estimates together with the triangle inequality give (2.19).

Finally, (2.20) follows by the inverse inequality in time and (2.19) with $s=1 / p+\alpha$, i.e.,

$$
\begin{aligned}
\left\|\left(U_{h}^{n}-u_{h}\left(t_{n}\right)\right)_{n=1}^{N}\right\|_{\ell^{\infty}\left(L^{2}(\Omega)\right)} & \leqslant c \tau^{-1 / p}\left\|\left(U_{h}^{n}-u_{h}\left(t_{n}\right)\right)_{n=1}^{N}\right\|_{\ell p\left(L^{2}(\Omega)\right)} \\
& \leqslant c \tau^{\alpha}\|g\|_{W^{1 / p+\alpha, p}\left(0, T ; L^{2}(\Omega)\right)}
\end{aligned}
$$

This completes the proof of the theorem.

REMARK 2.3 The estimate (2.19) is not sharp since, according to the proof, the restriction $s<1$ is only needed for $\alpha=1 / p^{\prime}$. Nonetheless, it is sufficient for the error analysis in Section 3.

\section{The optimal control problem and its numerical approximation}

In this section, we develop a numerical scheme for problem (1.1)-(1.2), and derive error bounds for the spatial and temporal discretizations.

\subsection{The continuous problem}

The first-order optimality condition of (1.1)-(1.2) was given in (Zhou \& Gong, 2016, Theorem 3.4).

THEOREM 3.1 Problem (1.1)-(1.2) admits a unique solution $q \in U_{\text {ad }}$. There exist a state $u \in L^{2}(0, T ; D(\Delta)) \cap$ $H_{L}^{\alpha}\left(0, T ; L^{2}(\Omega)\right)$ and an adjoint $z \in L^{2}(0, T ; D(\Delta)) \cap H_{R}^{\alpha}\left(0, T ; L^{2}(\Omega)\right)$ such that $(u, z, q)$ satisfies

$$
\begin{gathered}
{ }_{0} \partial_{t}^{\alpha} u-\Delta u=f+q, \quad 0<t \leqslant T, \quad \text { with } \quad u(0)=0, \\
{ }_{t} \partial_{T}^{\alpha} z-\Delta z=u-u_{d}, \quad 0 \leqslant t<T, \quad \text { with } \quad z(T)=0, \\
\quad(\gamma q+z, v-q)_{L^{2}\left(0, T ; L^{2}(\Omega)\right)} \geqslant 0, \quad \forall v \in U_{\mathrm{ad}} .
\end{gathered}
$$

where $(\cdot, \cdot)_{L^{2}\left(0, T ; L^{2}(\Omega)\right)}$ denotes the $L^{2}\left(0, T ; L^{2}(\Omega)\right)$ inner product.

Let $P_{U_{\text {ad }}}$ be the pointwise projection operator defined by

$$
P_{U_{\mathrm{ad}}}(q)=\max (a, \min (q, b)) .
$$

It is bounded on $W^{s, p}\left(0, T ; L^{2}(\Omega)\right)$ for $0 \leqslant s \leqslant 1$ and $1 \leqslant p \leqslant \infty$

$$
\left\|P_{U_{\text {ad }}} u\right\|_{W^{s, p}\left(0, T ; L^{2}(\Omega)\right)} \leqslant c\|u\|_{W^{s, p}\left(0, T ; L^{2}(\Omega)\right)} .
$$

This estimate holds trivially for $s=0$ and $s=1$ (Ziemer, 1989, Corollary 2.1.8), and the case $0<s<1$ follows by interpolation. Then (3.3) is equivalent to the complementarity condition

$$
q=P_{U_{\mathrm{ad}}}\left(-\gamma^{-1} z\right)
$$

Now we give higher temporal regularity of the triple $(u, z, q)$. Note that the constant $c$ depends on the scalar $\gamma$ (see also the proof of Lemma 3.4) and the constraint bounds $a$ and $b$.

Lemma 3.1 For any $s \in(0,1 / 2)$, let $u_{d} \in H^{s}\left(0, T ; L^{2}(\Omega)\right)$ and $f \in H^{s}\left(0, T ; L^{2}(\Omega)\right)$. Then the solution $(u, z, q)$ of problem (3.1)-(3.3) satisfies the following estimate

$$
\|q\|_{H^{\min (1, \alpha+s)}\left(0, T ; L^{2}(\Omega)\right)}+\|u\|_{H^{\alpha+s}\left(0, T ; L^{2}(\Omega)\right)}+\|z\|_{H^{\alpha+s}\left(0, T ; L^{2}(\Omega)\right)} \leqslant c .
$$

Proof. Let $r=\min (1, \alpha+s)$. By (3.6) and (3.5), we have

$$
\|q\|_{H^{r}\left(0, T ; L^{2}(\Omega)\right)} \leqslant c\|z\|_{H^{r}\left(0, T ; L^{2}(\Omega)\right)} \leqslant c\|z\|_{H^{\alpha+s}\left(0, T ; L^{2}(\Omega)\right)} .
$$


Applying Theorem 2.1 to (3.2) yields

$$
\|z\|_{H^{\alpha+s}\left(0, T ; L^{2}(\Omega)\right)} \leqslant c\left(\|u\|_{H^{s}\left(0, T ; L^{2}(\Omega)\right)}+\left\|u_{d}\right\|_{H^{s}\left(0, T ; L^{2}(\Omega)\right)}\right) \leqslant c\|u\|_{H^{s}\left(0, T ; L^{2}(\Omega)\right)}+c .
$$

Similarly, applying Theorem 2.1 to (3.1) gives

$$
\|u\|_{H^{\alpha+s}\left(0, T ; L^{2}(\Omega)\right)} \leqslant c\left(\|f\|_{H^{s}\left(0, T ; L^{2}(\Omega)\right)}+\|q\|_{H^{s}\left(0, T ; L^{2}(\Omega)\right)}\right) \leqslant c+c\|q\|_{H^{s}\left(0, T ; L^{2}(\Omega)\right)} .
$$

The last three estimates together imply

$$
\|q\|_{H^{r}\left(0, T ; L^{2}(\Omega)\right)} \leqslant c+c\|q\|_{H^{s}\left(0, T ; L^{2}(\Omega)\right)} \leqslant c+c_{\mathcal{E}^{\prime}}\|q\|_{L^{2}\left(0, T ; L^{2}(\Omega)\right)}+\varepsilon^{\prime}\|q\|_{H^{r}\left(0, T ; L^{2}(\Omega)\right)},
$$

where the last step is due to the interpolation inequality (Tartar, 2006, Lemma 24.1)

$$
\|q\|_{H^{s}\left(0, T ; L^{2}(\Omega)\right)} \leqslant c_{\mathcal{E}^{\prime}}\|q\|_{L^{2}\left(0, T ; L^{2}(\Omega)\right)}+\varepsilon^{\prime}\|q\|_{H^{r}\left(0, T ; L^{2}(\Omega)\right)} .
$$

By choosing a small $\varepsilon^{\prime}>0$ and the $L^{\infty}$ bounded of $q$, cf. (3.6), we obtain

$$
\|q\|_{H^{r}\left(0, T ; L^{2}(\Omega)\right)} \leqslant c+c_{\mathcal{E}^{\prime}}\|q\|_{L^{2}\left(0, T ; L^{2}(\Omega)\right)} \leqslant c .
$$

This shows the bound on $q$. (3.8) and (3.7) give the bound on $u$, and that of $z$ follows similarly.

Next, we give an improved stability estimate on $q$.

LEMMA 3.2 Let $p>1 / \alpha$ be sufficiently large so that $\alpha \in\left(0,1 / p^{\prime}\right), u_{d} \in W^{\alpha, p}\left(0, T ; L^{2}(\Omega)\right)$ and $f \in$ $L^{p}\left(0, T ; L^{2}(\Omega)\right)$. Then the optimal control $q$ satisfies:

$$
\|q\|_{W^{1 / p+\alpha-\varepsilon, p}\left(0, T ; L^{2}(\Omega)\right)} \leqslant c
$$

where the constant $c$ depends on $\left\|u_{d}\right\|_{W^{\alpha, p}\left(0, T ; L^{2}(\Omega)\right)}$ and $\|f\|_{L^{p}\left(0, T ; L^{2}(\Omega)\right)}$.

Proof. The condition $\alpha \in\left(0,1 / p^{\prime}\right)$ implies $r:=1 / p+\alpha-\varepsilon<1$. Thus (3.5) and Theorem 2.1 (with $s=r-\alpha)$ imply

$$
\|q\|_{W^{r, p}\left(0, T ; L^{2}(\Omega)\right)} \leqslant c\|z\|_{W^{r, p}\left(0, T ; L^{2}(\Omega)\right)} \leqslant c\left\|u-u_{d}\right\|_{W^{r-\alpha, p}\left(0, T ; L^{2}(\Omega)\right)},
$$

Since $p>1 / \alpha, r-\alpha=1 / p-\varepsilon<\alpha$ and thus Theorem 2.1 (with $s=0$ ) and (3.6) give

$$
\left\|u-u_{d}\right\|_{W^{r-\alpha, p}\left(0, T ; L^{2}(\Omega)\right)} \leqslant c\left\|u-u_{d}\right\|_{W^{\alpha, p}\left(0, T ; L^{2}(\Omega)\right)} \leqslant c\|f+q\|_{L^{p}\left(0, T ; L^{2}(\Omega)\right)}+c \leqslant c,
$$

where the second last inequality is due to Theorem 2.1 with $s=0$, and the last inequality due to the pointwise boundedness of $q$, cf. (3.6). The last two estimates together imply the desired result.

\subsection{Spatially semidiscrete scheme}

Now we give a spatially semidiscrete scheme for problem (1.1)-(1.2): find $q_{h} \in U_{\text {ad }}$ such that

$$
\min _{q_{h} \in U_{\mathrm{ad}}} J\left(u_{h}, q_{h}\right)=\frac{1}{2}\left\|u_{h}-u_{d}\right\|_{L^{2}\left(0, T ; L^{2}(\Omega)\right)}^{2}+\frac{\gamma}{2}\left\|q_{h}\right\|_{L^{2}\left(0, T ; L^{2}(\Omega)\right)}^{2},
$$

subject to the semidiscrete problem

$$
{ }_{0} \partial_{t}^{\alpha} u_{h}-\Delta_{h} u_{h}=P_{h}\left(f+q_{h}\right), \quad 0<t \leqslant T, \quad \text { with } \quad u_{h}(0)=0 .
$$

Note that in the scheme (3.9)-(3.10), the control variable $q_{h}$ is not discretized directly, but instead in a variational sense due to Hinze (2005). This choice greatly facilitates the analysis, while also leads to optimal order convergence rates. In passing, note that there are other possible strategies to discretize the control variable $q$, e.g., cellwise constant/linear approximation and postprocessing; see Meidner \& 
Vexler (2008) for the standard parabolic counterparts. It would be interesting to analyze these discretization strategies, which, however, is beyond the scope of the present work.

Similar to Theorem 3.1, problem (3.9)-(3.10) admits a unique solution $q_{h} \in U_{\text {ad }}$. The first-order optimality system reads:

$$
\begin{gathered}
{ }_{0} \partial_{t}^{\alpha} u_{h}-\Delta_{h} u_{h}=P_{h}\left(f+q_{h}\right), \quad 0<t \leqslant T, \quad \text { with } \quad u_{h}(0)=0, \\
{ }_{t} \partial_{T}^{\alpha} z_{h}-\Delta_{h} z_{h}=P_{h}\left(u_{h}-u_{d}\right), \quad 0 \leqslant t<T, \quad \text { with } \quad z_{h}(T)=0, \\
\quad\left(\gamma q_{h}+z_{h}, v-q_{h}\right)_{L^{2}\left(0, T ; L^{2}(\Omega)\right)} \geqslant 0, \quad \forall v \in U_{\mathrm{ad}} .
\end{gathered}
$$

The variational inequality (3.13) is equivalent to

$$
q_{h}=P_{U_{\mathrm{ad}}}\left(-\gamma^{-1} z_{h}\right) .
$$

For the scheme (3.11)-(3.13), we have the next error bound (Zhou \& Gong, 2016, Theorem 4.6).

THEOREM 3.2 For $f, u_{d} \in L^{2}\left(0, T ; L^{2}(\Omega)\right)$, let $(u, z, q)$ and $\left(u_{h}, z_{h}, q_{h}\right)$ be the solutions of problems (3.1)-(3.3) and (3.11)-(3.13), respectively. Then there hold

$$
\begin{array}{r}
\left\|u-u_{h}\right\|_{L^{2}\left(0, T ; L^{2}(\Omega)\right)}+\left\|z-z_{h}\right\|_{L^{2}\left(0, T ; L^{2}(\Omega)\right)}+\left\|q-q_{h}\right\|_{L^{2}\left(0, T ; L^{2}(\Omega)\right)} \leqslant c h^{2}, \\
\left\|\nabla\left(u-u_{h}\right)\right\|_{L^{2}\left(0, T ; L^{2}(\Omega)\right)}+\left\|\nabla\left(z-z_{h}\right)\right\|_{L^{2}\left(0, T ; L^{2}(\Omega)\right)} \leqslant c h .
\end{array}
$$

Next, we present the regularity of the semidiscrete solution $\left(u_{h}, z_{h}, q_{h}\right)$. The proof is similar to the continuous case in Lemmas 3.1 and 3.2 and hence omitted.

LEMMA 3.3 Let $s \in(0,1 / 2), u_{d} \in H^{s}\left(0, T ; L^{2}(\Omega)\right)$ and $f \in H^{s}\left(0, T ; L^{2}(\Omega)\right)$. Then the solution $\left(u_{h}, z_{h}, q_{h}\right)$ of problem (3.11)-(3.13) satisfies

$$
\left\|q_{h}\right\|_{H^{\min (1, \alpha+s)}\left(0, T ; L^{2}(\Omega)\right)}+\left\|u_{h}\right\|_{H^{\alpha+s}\left(0, T ; L^{2}(\Omega)\right)}+\left\|z_{h}\right\|_{H^{\alpha+s}\left(0, T ; L^{2}(\Omega)\right)} \leqslant c .
$$

Further, for $u_{d} \in W^{\alpha, p}\left(0, T ; L^{2}(\Omega)\right), f \in L^{p}\left(0, T ; L^{2}(\Omega)\right)$, with $p>1 / \alpha$ and $\alpha \in\left(0,1 / p^{\prime}\right)$, there holds

$$
\left\|q_{h}\right\|_{W^{1 / p+\alpha-\varepsilon, p}\left(0, T ; L^{2}(\Omega)\right)} \leqslant c .
$$

Last, we derive a pointwise-in-time error estimate.

THEOREM 3.3 For $f, u_{d} \in H^{1}\left(0, T ; L^{2}(\Omega)\right)$, let $(u, z, q)$ and $\left(u_{h}, z_{h}, q_{h}\right)$ be the solutions of problems (3.1)-(3.3) and (3.11)-(3.13), respectively. Then with $\ell_{h}=\log (2+1 / h)$, there holds

$$
\left\|u-u_{h}\right\|_{L^{\infty}\left(0, T ; L^{2}(\Omega)\right)}+\left\|z-z_{h}\right\|_{L^{\infty}\left(0, T ; L^{2}(\Omega)\right)}+\left\|q-q_{h}\right\|_{L^{\infty}\left(0, T ; L^{2}(\Omega)\right)} \leqslant c \ell_{h}^{2} h^{2},
$$

where the constant $c$ depends on $\|f\|_{H^{1}\left(0, T ; L^{2}(\Omega)\right)}$ and $\left\|u_{d}\right\|_{H^{1}\left(0, T ; L^{2}(\Omega)\right)}$.

Proof. We employ the splitting $u-u_{h}=\left(u-u_{h}(q)\right)+\left(u_{h}(q)-u_{h}\right):=\rho+\vartheta$, where $u_{h}(q) \in X_{h}$ solves

$$
\partial_{t}^{\alpha} u_{h}(q)-\Delta_{h} u_{h}(q)=P_{h}(f+q), \quad 0<t \leqslant T, \quad \text { with } \quad u_{h}(q)(0)=0 .
$$

Then $u_{h}(q)$ is the semidiscrete solution of (2.1) with $g=f+q$, and $\rho$ is the FEM error for the direct problem. By (Jin et al., 2015, Theorem 3.7) and Lemma 3.2, we have

$$
\|\rho\|_{L^{\infty}\left(0, T ; L^{2}(\Omega)\right)} \leqslant c \ell_{h}^{2} h^{2}\|f+q\|_{L^{\infty}\left(0, T ; L^{2}(\Omega)\right)} \leqslant c \ell_{h}^{2} h^{2} .
$$

Since $\vartheta$ satisfies $\partial_{t}^{\alpha} \vartheta-\Delta_{h} \vartheta=P_{h}\left(q-q_{h}\right)$, for $0<t \leqslant T$ with $\vartheta(0)=0,(2.13), L^{2}(\Omega)$-stability of $P_{h}$, the conditions (3.6) and (3.14), and the pointwise contractivity of $P_{U_{\text {ad }}}$ imply

$$
\begin{gathered}
\left\|_{0} \partial_{t}^{\alpha} \vartheta\right\|_{L^{p}\left(0, T ; L^{2}(\Omega)\right)} \leqslant\left\|P_{h}\left(q-q_{h}\right)\right\|_{L^{p}\left(0, T ; L^{2}(\Omega)\right)} \\
\leqslant c\left\|q-q_{h}\right\|_{L^{p}\left(0, T ; L^{2}(\Omega)\right)} \leqslant c\left\|z-z_{h}\right\|_{L^{p}\left(0, T ; L^{2}(\Omega)\right)} .
\end{gathered}
$$


Next, it follows from (3.2), (3.12) and the identity $P_{h} \Delta=\Delta_{h} R_{h}$ (with $R_{h}: H^{1}(\Omega) \rightarrow X_{h}$ being Ritz projection) that $w_{h}:=P_{h} z-z_{h}$ satisfies $w_{h}(T)=0$ and

$$
{ }_{t} \partial_{T}^{\alpha} w_{h}-\Delta_{h} w_{h}=P_{h} u-u_{h}-\Delta_{h}\left(P_{h} z-R_{h} z\right), \quad 0 \leqslant t<T,
$$

and thus by applying $\Delta_{h}^{-1}$ on both sides leads to

$$
{ }_{t} \partial_{T}^{\alpha} \Delta_{h}^{-1} w_{h}-\Delta_{h} \Delta_{h}^{-1} w_{h}=\Delta_{h}^{-1}\left(P_{h} u-u_{h}\right)-\left(P_{h} z-R_{h} z\right) .
$$

The maximal $L^{p}$ regularity (2.13) and triangle inequality imply

$$
\begin{aligned}
\left\|w_{h}\right\|_{L^{p}\left(0, T ; L^{2}(\Omega)\right)} & \leqslant c\left\|\Delta_{h}^{-1}\left(P_{h} u-u_{h}\right)-\left(P_{h} z-R_{h} z\right)\right\|_{L^{p}\left(0, T ; L^{2}(\Omega)\right)} \\
& \leqslant c\left\|P_{h} u-u_{h}\right\|_{L^{p}\left(0, T ; L^{2}(\Omega)\right)}+c\left\|P_{h} z-R_{h} z\right\|_{L^{p}\left(0, T ; L^{2}(\Omega)\right)}
\end{aligned}
$$

The $L^{2}(\Omega)$-stability of $P_{h}$ and triangle inequality yield

$$
\left\|P_{h} u-u_{h}\right\|_{L^{p}\left(0, T ; L^{2}(\Omega)\right)} \leqslant c\left\|u-u_{h}\right\|_{L^{p}\left(0, T ; L^{2}(\Omega)\right)} \leqslant c\left(\|\vartheta\|_{L^{p}\left(0, T ; L^{2}(\Omega)\right)}+\|\rho\|_{L^{p}\left(0, T ; L^{2}(\Omega)\right)}\right),
$$

and Theorem 2.1 (with $s=0$ ) and lemma 3.3 give

$$
\left\|P_{h} z-R_{h} z\right\|_{L^{p}\left(0, T ; L^{2}(\Omega)\right)} \leqslant c\|z\|_{L^{p}\left(0, T ; H^{2}(\Omega)\right)} h^{2} \leqslant c\left\|u-u_{d}\right\|_{L^{p}\left(0, T ; L^{2}(\Omega)\right)} h^{2} \leqslant c h^{2} .
$$

The last three estimates and (3.15) yield

$$
\left\|w_{h}\right\|_{L^{p}\left(0, T ; L^{2}(\Omega)\right)} \leqslant c\|\vartheta\|_{L^{p}\left(0, T ; L^{2}(\Omega)\right)}+c \ell_{h}^{2} h^{2} .
$$

Thus repeating the preceding argument yields

$$
\begin{aligned}
\left\|z-z_{h}\right\|_{L^{p}\left(0, T ; L^{2}(\Omega)\right)} & \leqslant\left\|z-P_{h} z\right\|_{L^{p}\left(0, T ; L^{2}(\Omega)\right)}+\left\|w_{h}\right\|_{L^{p}\left(0, T ; L^{2}(\Omega)\right)} \\
& \leqslant c\|\vartheta\|_{L^{p}\left(0, T ; L^{2}(\Omega)\right)}+c \ell_{h}^{2} h^{2} .
\end{aligned}
$$

Substituting this estimate into (3.16) and by Sobolev embedding $W^{\alpha, p}\left(0, T ; L^{2}(\Omega)\right) \hookrightarrow L^{p_{\alpha}}\left(0, T ; L^{2}(\Omega)\right)$, with the critical exponent $p_{\alpha}=p /(1-p \alpha)$ if $p \alpha<1$, and $p_{\alpha}=\infty$ if $p \alpha>1$ :

$$
\|\vartheta\|_{L^{p \alpha}\left(0, T ; L^{2}(\Omega)\right)} \leqslant c\left\|_{0} \partial_{t}^{\alpha} \vartheta\right\|_{L^{p}\left(0, T ; L^{2}(\Omega)\right)} \leqslant c\|\vartheta\|_{L^{p}\left(0, T ; L^{2}(\Omega)\right)}+c \ell_{h}^{2} h^{2} .
$$

A finite number of repeated applications of this inequality yields

$$
\|\vartheta\|_{L^{\infty}\left(0, T ; L^{2}(\Omega)\right)} \leqslant c\|\vartheta\|_{L^{2}\left(0, T ; L^{2}(\Omega)\right)}+c \ell_{h}^{2} h^{2} \leqslant c \ell_{h}^{2} h^{2}
$$

where we have used the fact that, by maximal $L^{p}$ regularity (2.13) and Theorem 3.2,

$$
\|\vartheta\|_{L^{2}\left(0, T ; L^{2}(\Omega)\right)}=\left\|u_{h}(q)-u_{h}\right\|_{L^{2}\left(0, T ; L^{2}(\Omega)\right)} \leqslant c\left\|q-q_{h}\right\|_{L^{2}\left(0, T ; L^{2}(\Omega)\right)} \leqslant c h^{2} .
$$

This gives the desired bound on $\left\|u-u_{h}\right\|_{L^{\infty}\left(0, T ; L^{2}(\Omega)\right)}$. The bounds on $\left\|z-z_{h}\right\|_{L^{\infty}\left(0, T ; L^{2}(\Omega)\right)}$ and $\| q-$ $q_{h} \|_{L^{\infty}\left(0, T ; L^{2}(\Omega)\right)}$ follow similarly (and also by the contraction property of $P_{U_{\mathrm{ad}}}$ ).

REMARK 3.1 By the best approximation properties for piecewise linear finite element spaces, the error estimates in Theorem 3.3 are of optimal order, up to the logarithmic factor $\ell_{h}^{2}$. The latter factor is also present for the direct problem with a source $f \in L^{\infty}\left(0, T ; L^{2}(\Omega)\right)$ (Jin et al., 2015, Theorem 3.7). 


\subsection{Fully discrete scheme}

Now we turn to the fully discrete approximation of (1.1)-(1.2), with L1 scheme or BE-CQ time stepping (and variational discretization for the control variable). First, we define a time-discrete admissible set

$$
U_{a d}^{\tau}=\left\{\vec{Q}_{h}=\left(Q_{h}^{n-1}\right)_{n=1}^{N} \in L^{2}(\Omega)^{N}: a \leqslant Q_{h}^{n-1} \leqslant b, n=1,2, \ldots, N\right\},
$$

and consider the following fully discrete problem:

$$
\min _{\vec{Q}_{h} \in U_{a d}^{\tau}} \frac{\tau}{2} \sum_{n=1}^{N}\left(\left\|U_{h}^{n}-u_{d}^{n}\right\|_{L^{2}(\Omega)}^{2}+\gamma\left\|Q_{h}^{n-1}\right\|_{L^{2}(\Omega)}^{2}\right),
$$

subject to the fully discrete problem

$$
{ }_{0} \bar{\partial}_{\tau}^{\alpha} U_{h}^{n}-\Delta_{h} U_{h}^{n}=f_{h}^{n}+P_{h} Q_{h}^{n-1}, \quad n=1,2, \ldots, N, \quad \text { with } U_{h}^{0}=0,
$$

with $u_{d}^{n}=u_{d}\left(t_{n}\right)$ and $f_{h}^{n}=P_{h} f\left(t_{n}\right)$. Note that like the semidiscrete case, the admissible set $U_{\text {ad }}$ is not directly discretized in space, but only in a variational sense. Let $\bar{\partial}_{\tau}^{\alpha} \varphi^{n}$ be the L1/BE-CQ approximation of the right-sided Riemann-Liouville fractional derivative ${ }_{t} \partial_{T}^{\alpha} \varphi\left(t_{n}\right)$ :

$$
\bar{\partial}_{\tau}^{\alpha} \varphi^{N-n}=\tau^{-\alpha} \sum_{j=0}^{n} \beta_{n-j} \varphi^{N-j} .
$$

Then the fully discrete problem is to find $\left(U_{h}^{n}, Z_{h}^{n}, Q_{h}^{n}\right)$ such that (Zhou \& Gong, 2016, Section 5)

$$
\begin{aligned}
{ }_{0} \bar{\partial}_{\tau}^{\alpha} U_{h}^{n}-\Delta_{h} U_{h}^{n} & =f_{h}^{n}+P_{h} Q_{h}^{n-1}, & & n=1,2, \ldots, N, \quad \text { with } U_{h}^{0}=0, \\
\bar{\partial}_{\tau}^{\alpha} Z_{h}^{n-1}-\Delta_{h} Z_{h}^{n-1} & =U_{h}^{n}-P_{h} u_{d}^{n}, & & n=1,2, \ldots, N, \quad \text { with } Z_{h}^{N}=0, \\
\left(\gamma Q_{h}^{n-1}+Z_{h}^{n-1}, v-Q_{h}^{n-1}\right) & \geqslant 0, & & \forall v \in L^{2}(\Omega) \text { s.t. } a \leqslant v \leqslant b .
\end{aligned}
$$

Similar to (3.13), (3.22) can be rewritten as

$$
Q_{h}^{n-1}=P_{U_{a d}}\left(-\gamma^{-1} Z_{h}^{n-1}\right), \quad n=1,2, \ldots, N .
$$

To simplify the notation, we denote the $\ell^{2}\left(L^{2}(\Omega)\right)$ inner product by

$$
[\mathbf{v}, \mathbf{w}]_{\tau}=\tau \sum_{n=1}^{N}\left(v_{n}, w_{n}\right) \quad \forall \mathbf{v}=\left(v_{n}\right)_{n=1}^{N}, \mathbf{w}=\left(w_{n}\right)_{n=1}^{N} \in L^{2}(\Omega)^{N},
$$

and we shall identify vectors with sequences below. Let ${ }_{0} \bar{\partial}_{\tau}^{\alpha} \mathbf{v}=\left({ }_{0} \bar{\partial}_{\tau}^{\alpha} v_{h}^{n}\right)_{n=1}^{N} \in L^{2}(\Omega)^{N}$ and $\bar{\partial}_{\tau}^{\alpha} \mathbf{w}=$ $\left(\bar{\partial}_{\tau}^{\alpha} w_{h}^{n-1}\right)_{n=1}^{N} \in L^{2}(\Omega)^{N}$. Then the following identity holds (Zhou \& Gong, 2016, Section 5.2)

$$
\left.{ }_{0} \bar{\partial}_{\tau}^{\alpha} \mathbf{v}, \mathbf{w}\right]_{\tau}=\left[\mathbf{v}, \bar{\partial}_{\tau}^{\alpha} \mathbf{w}\right]_{\tau} \quad \forall \mathbf{v}, \mathbf{w} \in L^{2}(\Omega)^{N}
$$

Thus, $\bar{\partial}_{\tau}^{\alpha}$ is the adjoint to ${ }_{0} \bar{\partial}_{\tau}^{\alpha}$ with respect to $[\cdot, \cdot]_{\tau}$. Let

$$
\begin{array}{lll}
\mathbf{U}_{h}=\left(U_{h}^{n}\right)_{n=1}^{N}, & \mathbf{Z}_{h}=\left(Z_{h}^{n-1}\right)_{n=1}^{N}, & \mathbf{Q}_{h}=\left(Q_{h}^{n-1}\right)_{n=1}^{N}, \\
\mathbf{u}_{h}=\left(u_{h}\left(t_{n}\right)\right)_{n=1}^{N}, & \mathbf{z}_{h}=\left(z_{h}\left(t_{n-1}\right)\right)_{n=1}^{N}, & \mathbf{q}_{h}=\left(q_{h}\left(t_{n-1}\right)\right)_{n=1}^{N} .
\end{array}
$$

Next we introduce two auxiliary problems. Let $\left.\mathbf{U}_{h}\left(q_{h}\right)=\left(U_{h}^{n}\left(q_{h}\right)\right)\right)_{n=1}^{N} \in X_{h}^{N}$ solve

$$
{ }_{0} \bar{\partial}_{\tau}^{\alpha} U_{h}^{n}\left(q_{h}\right)-\Delta_{h} U_{h}^{n}\left(q_{h}\right)=f_{h}^{n}+q_{h}\left(t_{n-1}\right), \quad n=1, \ldots, N, \quad \text { with } U_{h}^{0}\left(q_{h}\right)=0 .
$$

By Lemma 3.3, the pointwise evaluation $q_{h}\left(t_{n}\right)$ does make sense, and thus problem (3.25) is well defined. For any $\mathbf{v}_{h}=\left(v_{h}^{n}\right)_{n=1}^{N} \in X_{h}^{N}$, let $\mathbf{Z}_{h}\left(\mathbf{v}_{h}\right)=\left(Z_{h}^{n-1}\left(\mathbf{v}_{h}\right)\right)_{n=1}^{N} \in X_{h}^{N}$ solve

$$
\bar{\partial}_{\tau}^{\alpha} Z_{h}^{n-1}\left(\mathbf{v}_{h}\right)-\Delta_{h} Z_{h}^{n-1}\left(\mathbf{v}_{h}\right)=v_{h}^{n}-P_{h} u_{d}^{n}, n=1,2, \ldots, N \text {, with } Z_{h}^{N}\left(\mathbf{v}_{h}\right)=0 .
$$

The rest of this part is devoted to error analysis. First, we bound $\left\|\mathbf{q}_{h}-\mathbf{Q}_{h}\right\|_{\ell^{2}\left(L^{2}(\Omega)\right)}$. 
Lemma 3.4 For $\mathbf{Q}_{h}, \mathbf{q}_{h}, \mathbf{Z}_{h}$ and $\mathbf{Z}_{h}\left(\mathbf{U}_{h}\left(q_{h}\right)\right)$ defined as above, there holds

$$
\gamma\left\|\mathbf{Q}_{h}-\mathbf{q}_{h}\right\|_{\ell^{2}\left(L^{2}(\Omega)\right)}^{2} \leqslant\left[\mathbf{q}_{h}-\mathbf{Q}_{h}, \mathbf{Z}_{h}\left(\mathbf{U}_{h}\left(q_{h}\right)\right)-\mathbf{z}_{h}\right]_{\tau} .
$$

Proof. It follows from (3.20) and (3.25), similarly from (3.21) and (3.26), that

$$
\left({ }_{0} \bar{\partial}_{\tau}^{\alpha}-\Delta_{h}\right)\left(\mathbf{U}_{h}\left(q_{h}\right)-\mathbf{U}_{h}\right)=\mathbf{q}_{h}-\mathbf{Q}_{h} \quad \text { and } \quad\left(\bar{\partial}_{\tau}^{\alpha}-\Delta_{h}\right)\left(\mathbf{Z}_{h}\left(\mathbf{v}_{h}\right)-\mathbf{Z}_{h}\right)=\mathbf{v}_{h}-\mathbf{U}_{h} .
$$

Together with (3.24), these identities imply

$$
\begin{aligned}
{\left[\mathbf{q}_{h}-\mathbf{Q}_{h}, \mathbf{Z}_{h}-\mathbf{Z}_{h}\left(\mathbf{U}_{h}\left(q_{h}\right)\right)\right]_{\tau} } & =\left[\left({ }_{0} \bar{\partial}_{\tau}^{\alpha}-\Delta_{h}\right)\left(\mathbf{U}_{h}\left(q_{h}\right)-\mathbf{U}_{h}\right), \mathbf{Z}_{h}-\mathbf{Z}_{h}\left(\mathbf{U}_{h}\left(q_{h}\right)\right)\right]_{\tau} \\
& =\left[\mathbf{U}_{h}\left(q_{h}\right)-\mathbf{U}_{h},\left(\bar{\partial}_{\tau}^{\alpha}-\Delta_{h}\right)\left(\mathbf{Z}_{h}-\mathbf{Z}_{h}\left(\mathbf{U}_{h}\left(q_{h}\right)\right)\right)\right]_{\tau} \\
& =-\left\|\mathbf{U}_{h}\left(q_{h}\right)-\mathbf{U}_{h}\right\|_{\ell^{2}\left(L^{2}(\Omega)\right)}^{2} \leqslant 0 .
\end{aligned}
$$

Next, since (3.14) holds pointwise in time, i.e., $q_{h}\left(t_{n-1}\right)=P_{U_{\text {ad }}}\left(-\gamma^{-1} z_{h}\left(t_{n-1}\right)\right)$, we have

$$
\left(q_{h}\left(t_{n-1}\right)+\gamma^{-1} z_{h}\left(t_{n-1}\right), \chi-q_{h}\left(t_{n-1}\right)\right) \geqslant 0, \quad \forall \chi \in L^{2}(\Omega) \text { s.t. } a \leqslant \chi \leqslant b .
$$

Upon setting $v=q_{h}\left(t_{n-1}\right)$ in (3.22) and $\chi=Q_{h}^{n-1}$ in (3.28), we deduce

$$
\begin{aligned}
\gamma\left\|\mathbf{Q}_{h}-\mathbf{q}_{h}\right\|_{\ell^{2}\left(L^{2}(\Omega)\right)}^{2} & =\gamma\left[\mathbf{Q}_{h}-\mathbf{q}_{h}, \mathbf{Q}_{h}\right]_{\tau}-\gamma\left[\mathbf{Q}_{h}-\mathbf{q}_{h}, \mathbf{q}_{h}\right]_{\tau} \\
& \leqslant\left[\mathbf{q}_{h}-\mathbf{Q}_{h}, \mathbf{Z}_{h}\right]_{\tau}-\left[\mathbf{q}_{h}-\mathbf{Q}_{h}, \mathbf{z}_{h}\right]_{\tau} \\
& =\left[\mathbf{q}_{h}-\mathbf{Q}_{h}, \mathbf{Z}_{h}-\mathbf{Z}_{h}\left(\mathbf{U}_{h}\left(q_{h}\right)\right)\right]_{\tau}+\left[\mathbf{q}_{h}-\mathbf{Q}_{h}, \mathbf{Z}_{h}\left(\mathbf{U}_{h}\left(q_{h}\right)\right)-\mathbf{z}_{h}\right]_{\tau} .
\end{aligned}
$$

Now invoking (3.27) completes the proof of the lemma.

The next result gives an error estimate for the approximate state $\mathbf{U}_{h}\left(q_{h}\right)$.

LEMmA 3.5 Let $f, u_{d} \in H^{1}\left(0, T ; L^{2}(\Omega)\right)$. For any $\varepsilon \in(0, \min (1 / 2, \alpha))$, there holds

$$
\left\|\mathbf{U}_{h}\left(q_{h}\right)-\mathbf{u}_{h}\right\|_{\ell^{2}\left(L^{2}(\Omega)\right)} \leqslant c \tau^{1 / 2+\min (1 / 2, \alpha-\varepsilon)} .
$$

Proof. By the triangle inequality, we have

$$
\left\|\mathbf{U}_{h}\left(q_{h}\right)-\mathbf{u}_{h}\right\|_{\ell^{2}\left(L^{2}(\Omega)\right)} \leqslant\left\|\mathbf{U}_{h}\left(q_{h}\right)-\widetilde{\mathbf{U}}_{h}\left(q_{h}\right)\right\|_{\ell^{2}\left(L^{2}(\Omega)\right)}+\left\|\widetilde{\mathbf{U}}_{h}\left(q_{h}\right)-\mathbf{u}_{h}\right\|_{\ell^{2}\left(L^{2}(\Omega)\right)},
$$

where $\widetilde{\mathbf{U}}_{h}\left(q_{h}\right)=\left(\widetilde{U}_{h}^{n}\left(q_{h}\right)\right)_{n=1}^{N}$ is the solution to

$$
{ }_{0} \bar{\partial}_{\tau}^{\alpha} \widetilde{U}_{h}^{n}\left(q_{h}\right)-\Delta_{h}^{n} \widetilde{U}_{h}^{n}\left(q_{h}\right)=f_{h}^{n}+\tilde{q}_{h}^{n}, \quad n=1,2, \ldots, N \quad \text { with } \widetilde{U}_{h}^{0}\left(q_{h}\right)=0,
$$

with $\tilde{q}_{h}^{n}=P_{h} q_{h}\left(t_{n}\right)$ (and $\left.\tilde{\mathbf{q}}_{h}=\left(\tilde{q}_{h}^{n}\right)_{n=1}^{N}\right)$. That is, $\widetilde{U}_{h}^{n}\left(q_{h}\right)$ is the fully discrete solution of problem (2.10) with $g=f+q_{h}$. By Lemmas 2.3 and 2.4, we have

$$
\left\|\mathbf{U}_{h}\left(q_{h}\right)-\widetilde{\mathbf{U}}_{h}\left(q_{h}\right)\right\|_{\ell^{2}\left(L^{2}(\Omega)\right)} \leqslant c\left\|\mathbf{q}_{h}-\tilde{\mathbf{q}}_{h}\right\|_{\ell^{2}\left(L^{2}(\Omega)\right)} \leqslant c \tau^{\min (1 / 2+\alpha-\varepsilon, 1)}\left\|q_{h}\right\|_{H^{1 / 2+\alpha-\varepsilon}\left(0, T ; L^{2}(\Omega)\right)} .
$$

Further, Theorem 2.3 (with $s=\min (1,1 / 2+\alpha-\varepsilon) \in(1 / 2,1)$ ) implies

$$
\begin{aligned}
\left\|\widetilde{\mathbf{U}}_{h}\left(q_{h}\right)-\mathbf{u}_{h}\right\|_{\ell^{2}\left(L^{2}(\Omega)\right)} & \leqslant c\left\|P_{h} f+q_{h}\right\|_{H^{s}\left(0, T ; L^{2}(\Omega)\right)} \tau^{s} \\
& \leqslant c\left(\left\|P_{h} f\right\|_{H^{s}\left(0, T ; L^{2}(\Omega)\right)}+\left\|q_{h}\right\|_{H^{s}\left(0, T ; L^{2}(\Omega)\right)}\right) \tau^{s} .
\end{aligned}
$$

The last two estimates and Lemma 3.3 (with $s=1 / 2-\varepsilon$ ) yield the desired assertion.

Now we can give an $\ell^{2}\left(L^{2}(\Omega)\right)$ error estimate for the approximation $\left(U_{h}^{n}, Z_{h}^{n}, Q_{h}^{n}\right)$. 
THEOREM 3.4 For $f \in H^{1}\left(0, T ; L^{2}(\Omega)\right)$ and $u_{d} \in H^{1}\left(0, T ; L^{2}(\Omega)\right),\left(u_{h}, z_{h}, q_{h}\right)$ and $\left(U_{h}^{n}, Z_{h}^{n}, Q_{h}^{n}\right)$ be the solutions of problems (3.11)-(3.13) and (3.20)-(3.22), respectively. Then there holds for any small $\varepsilon>0$

$$
\left\|\mathbf{u}_{h}-\mathbf{U}_{h}\right\|_{\ell^{2}\left(L^{2}(\Omega)\right)}+\left\|\mathbf{z}_{h}-\mathbf{Z}_{h}\right\|_{\ell^{2}\left(L^{2}(\Omega)\right)}+\left\|\mathbf{q}_{h}-\mathbf{Q}_{h}\right\|_{\ell^{2}\left(L^{2}(\Omega)\right)} \leqslant c \tau^{1 / 2+\min (1 / 2, \alpha-\varepsilon)},
$$

where the constant $c$ depends on $\|f\|_{H^{1}\left(0, T ; L^{2}(\Omega)\right)}$ and $\left\|u_{d}\right\|_{H^{1}\left(0, T ; L^{2}(\Omega)\right)}$.

Proof. By Lemma 3.4 and the triangle inequality, we deduce

$$
\left\|\mathbf{Q}_{h}-\mathbf{q}_{h}\right\|_{\ell^{2}\left(L^{2}(\Omega)\right)} \leqslant c\left\|\mathbf{Z}_{h}\left(\mathbf{U}_{h}\left(q_{h}\right)\right)-\mathbf{Z}_{h}\left(\mathbf{u}_{h}\right)\right\|_{\ell^{2}\left(L^{2}(\Omega)\right)}+c\left\|\mathbf{Z}_{h}\left(\mathbf{u}_{h}\right)-\mathbf{z}_{h}\right\|_{\ell^{2}\left(L^{2}(\Omega)\right)} .
$$

It suffices to bound the two terms on the right hand side. Lemmas 2.3 and 3.5 imply

$$
\left\|\mathbf{Z}_{h}\left(\mathbf{U}_{h}\left(q_{h}\right)\right)-\mathbf{Z}_{h}\left(\mathbf{u}_{h}\right)\right\|_{\ell^{2}\left(L^{2}(\Omega)\right)} \leqslant c\left\|\mathbf{U}_{h}\left(q_{h}\right)-\mathbf{u}_{h}\right\|_{\ell^{2}\left(L^{2}(\Omega)\right)} \leqslant c \tau^{r} .
$$

with $r=1 / 2+\min (1 / 2, \alpha-\varepsilon)$. Further, since $\mathbf{Z}_{h}\left(\mathbf{u}_{h}\right)$ is a fully discrete approximation to $z_{h}\left(u_{h}\right)$, by Theorem 2.3 (with $s=r$ ) and Lemma 3.3, we have

$$
\left\|\mathbf{Z}_{h}\left(\mathbf{u}_{h}\right)-\mathbf{z}_{h}\right\|_{\ell^{2}\left(L^{2}(\Omega)\right)} \leqslant c\left\|u_{h}-P_{h} u_{d}\right\|_{H^{r}\left(0, T ; L^{2}(\Omega)\right)} \tau^{r} \leqslant c \tau^{r} .
$$

Thus, we obtain the estimate $\left\|\mathbf{Q}_{h}-\mathbf{q}_{h}\right\|_{\ell^{2}\left(L^{2}(\Omega)\right)} \leqslant c \tau^{r}$. Next, by Lemmas 2.3 and 3.5, we deduce

$$
\begin{aligned}
\left\|\mathbf{U}_{h}-\mathbf{u}_{h}\right\|_{\ell^{2}\left(L^{2}(\Omega)\right)} & \leqslant\left\|\mathbf{U}_{h}-\mathbf{U}_{h}\left(\mathbf{q}_{h}\right)\right\|_{\ell^{2}\left(L^{2}(\Omega)\right)}+\left\|\mathbf{U}_{h}\left(\mathbf{q}_{h}\right)-\mathbf{u}_{h}\right\|_{\ell^{2}\left(L^{2}(\Omega)\right)} \\
& \leqslant c\left\|\mathbf{Q}_{h}-\mathbf{q}_{h}\right\|_{\ell^{2}\left(L^{2}(\Omega)\right)}+\left\|\mathbf{U}_{h}\left(\mathbf{q}_{h}\right)-\mathbf{u}_{h}\right\|_{\ell^{2}\left(L^{2}(\Omega)\right)} \leqslant c \tau^{r} .
\end{aligned}
$$

Similarly, $\left\|\mathbf{Z}_{h}-\mathbf{z}_{h}\right\|_{\ell^{2}\left(L^{2}(\Omega)\right)}$ can be bounded by

$$
\begin{aligned}
\left\|\mathbf{Z}_{h}-\mathbf{z}_{h}\right\|_{\ell^{2}\left(L^{2}(\Omega)\right)} & \leqslant\left\|\mathbf{Z}_{h}-\mathbf{Z}_{h}\left(\mathbf{u}_{h}\right)\right\|_{\ell^{2}\left(L^{2}(\Omega)\right)}+\left\|\mathbf{Z}_{h}\left(\mathbf{u}_{h}\right)-\mathbf{z}_{h}\right\|_{\ell^{2}\left(L^{2}(\Omega)\right)} \\
& \leqslant c\left\|\mathbf{U}_{h}-\mathbf{u}_{h}\right\|_{\ell^{2}\left(L^{2}(\Omega)\right)}+\left\|\mathbf{Z}_{h}\left(\mathbf{u}_{h}\right)-\mathbf{z}_{h}\right\|_{\ell^{2}\left(L^{2}(\Omega)\right)} \leqslant c \tau^{r} .
\end{aligned}
$$

This completes the proof of Theorem 3.4.

Last, we give a pointwise-in-time error estimate for the approximation $\left(U_{h}^{n}, Q_{h}^{n}, Z_{h}^{n}\right)$.

THEOREM 3.5 For $f, u_{d} \in W^{1, p}\left(0, T ; L^{2}(\Omega)\right) \cap H^{1}\left(0, T ; L^{2}(\Omega)\right), p>1 / \alpha$ with $\alpha \in\left(0,1 / p^{\prime}\right)$, let $\left(u_{h}, z_{h}, q_{h}\right)$ and $\left(U_{h}^{n}, Z_{h}^{n}, Q_{h}^{n}\right)$ be the solutions of problems (3.11)-(3.13) and (3.20)-(3.22), respectively. Then there holds for any small $\varepsilon>0$

$$
\max _{1 \leqslant n \leqslant N}\left(\left\|u_{h}^{n}-U_{h}^{n}\right\|_{L^{2}(\Omega)}+\left\|z_{h}^{n-1}-Z_{h}^{n-1}\right\|_{L^{2}(\Omega)}+\left\|q_{h}^{n-1}-Q_{h}^{n-1}\right\|_{L^{2}(\Omega)}\right) \leqslant c \tau^{\alpha-\varepsilon},
$$



Proof. It follows from (3.20) and (3.25) that $U_{h}^{0}-U_{h}^{0}\left(q_{h}\right)=0$ and

$$
{ }_{0} \bar{\partial}_{\tau}^{\alpha}\left(U_{h}^{n}-U_{h}^{n}\left(q_{h}\right)\right)-\Delta_{h}\left(U_{h}^{n}-U_{h}^{n}\left(q_{h}\right)\right)=Q_{h}^{n-1}-q_{h}\left(t_{n-1}\right), \quad n=1, \ldots, N .
$$

By Lemma 2.3 and the inverse inequality (in time), we obtain for any $1 / \alpha<p_{1}<\infty$

$$
\begin{aligned}
\left\|_{0} \bar{\partial}_{\tau}^{\alpha}\left(U_{h}^{n}-U_{h}^{n}\left(q_{h}\right)\right)_{n=1}^{N}\right\|_{\ell^{p_{1}}\left(L^{2}(\Omega)\right)} & \leqslant c\left\|\left(Q_{h}^{n-1}-q_{h}\left(t_{n-1}\right)\right)_{n=1}^{N}\right\|_{\ell^{p_{1}}\left(L^{2}(\Omega)\right)} \\
& \leqslant c \tau^{\min \left(0,1 / p_{1}-1 / 2\right)}\left\|\left(Q_{h}^{n-1}-q_{h}\left(t_{n-1}\right)\right)_{n=1}^{N}\right\|_{\ell^{2}\left(L^{2}(\Omega)\right)} .
\end{aligned}
$$

This and Theorem 3.4 imply

$$
\left\|_{0} \bar{\partial}_{\tau}^{\alpha}\left(U_{h}^{n}-U_{h}^{n}\left(q_{h}\right)\right)_{n=1}^{N}\right\|_{\ell^{p_{1}\left(L^{2}(\Omega)\right)}} \leqslant c \tau^{\min \left(1 / p_{1}, 1 / 2\right)+\min (1 / 2, \alpha-\varepsilon)} .
$$


By choosing $p_{1}>1 / \alpha$ sufficiently close to $1 / \alpha$ and discrete embedding (Jin et al. (2018b)),

$$
\begin{aligned}
\left\|\left(U_{h}^{n}-U_{h}^{n}\left(q_{h}\right)\right)_{n=1}^{N}\right\|_{\ell^{\infty}\left(L^{2}(\Omega)\right)} & \leqslant c\left\|_{0} \bar{\partial}_{\tau}^{\alpha}\left(U_{h}^{n}-U_{h}^{n}\left(q_{h}\right)\right)_{n=1}^{N}\right\|_{\ell^{p_{1}}\left(L^{2}(\Omega)\right)} \\
& \leqslant c \tau^{\min \left(1 / p_{1}, 1 / 2\right)+\min (1 / 2, \alpha-\varepsilon)} \leqslant c \tau^{\alpha-\varepsilon},
\end{aligned}
$$

where the last inequality follows from the inequality $\min \left(1 / p_{1}, 1 / 2\right)+\min (1 / 2, \alpha-\varepsilon) \geqslant \alpha-\varepsilon$, due to the choice of $p_{1}$. Further, by the definition of $\widetilde{U}_{h}^{n}\left(q_{h}\right)$ in (3.29), choosing $p_{2}>1 / \alpha$ sufficiently large so that $\alpha \in\left(0,1 / p_{2}^{\prime}\right)$ and applying (2.20) and Lemma 3.3, we get

$$
\begin{aligned}
\left\|u_{h}\left(t_{n}\right)-\widetilde{U}_{h}^{n}\left(q_{h}\right)\right\|_{L^{2}(\Omega)} & \leqslant c \tau^{\alpha-\varepsilon}\left\|f+q_{h}\right\|_{W^{1 / p_{2}+\alpha-\varepsilon, p_{2}\left(0, T ; L^{2}(\Omega)\right)}} \\
& \leqslant c \tau^{\alpha-\varepsilon}\left(\left\|q_{h}\right\|_{W^{1 / p_{2}+\alpha-\varepsilon, p_{2}}\left(0, T ; L^{2}(\Omega)\right)}+c\right) \leqslant c \tau^{\alpha-\varepsilon} .
\end{aligned}
$$

Last, by choosing $p_{3}>1 / \alpha$ sufficiently close to $1 / \alpha$, Lemmas $2.3,3.3$, and 2.4 , and discrete embedding (Jin et al. (2018b)), we obtain

$$
\begin{aligned}
& \left\|\left(\widetilde{U}_{h}^{n}\left(q_{h}\right)-U_{h}^{n}\left(q_{h}\right)\right)_{n=1}^{N}\right\|_{\ell^{\infty}\left(L^{2}(\Omega)\right)} \leqslant c\left\|_{0} \bar{\partial}_{\tau}^{\alpha}\left(\widetilde{U}_{h}^{n}\left(q_{h}\right)-U_{h}^{n}\left(q_{h}\right)\right)_{n=1}^{N}\right\|_{\ell^{p_{3}}\left(L^{2}(\Omega)\right)} \\
& \leqslant c\left\|\left(q_{h}\left(t_{n-1}\right)-q_{h}\left(t_{n}\right)\right)_{n=1}^{N}\right\|_{\ell^{p_{3}\left(L^{2}(\Omega)\right)}} \leqslant c \tau^{\alpha-\varepsilon} .
\end{aligned}
$$

The last three estimates yield the desired bound on $\left\|u_{h}\left(t_{n}\right)-U_{h}^{n}\right\|_{L^{2}(\Omega)}$. The bound on $\| z_{h}\left(t_{n-1}\right)-$ $Z_{h}^{n-1} \|_{L^{2}(\Omega)}$ follows similarly, and that on $\left\|q_{h}\left(t_{n-1}\right)-Q_{h}^{n-1}\right\|_{L^{2}(\Omega)}$ by the contraction property of $P_{U_{\mathrm{ad}}}$.

REMARK 3.2 Theorem 3.5 gives an $O\left(\tau^{\alpha-\varepsilon}\right)$ convergence rate in the $\ell^{\infty}\left(L^{2}(\Omega)\right)$-norm for the time discretization errors of the control, state and adjoint variables. This estimate agrees with both the numerical experiments in Section 4 and the regularity result in Lemma 3.3. Indeed, Lemma 3.3 implies

$$
\begin{aligned}
& \left\|u_{h}\right\|_{C^{\alpha-\varepsilon}\left([0, T] ; L^{2}(\Omega)\right)}+\left\|z_{h}\right\|_{C^{\alpha-\varepsilon}\left([0, T] ; L^{2}(\Omega)\right)}+\left\|q_{h}\right\|_{C^{\alpha-\varepsilon}\left([0, T] ; L^{2}(\Omega)\right)} \\
\leqslant & c\left(\left\|u_{h}\right\|_{H^{\alpha+1 / 2-\varepsilon}\left(0, T ; L^{2}(\Omega)\right)}+\left\|z_{h}\right\|_{H^{\alpha+1 / 2-\varepsilon}\left(0, T ; L^{2}(\Omega)\right)}+\left\|q_{h}\right\|_{W^{\alpha+1 / p-\varepsilon, p}\left(0, T ; L^{2}(\Omega)\right)}\right) \leqslant c .
\end{aligned}
$$

Since the $O\left(\tau^{\alpha}\right)$ convergence rate (uniform in time) is optimal for the direct problem (Jin et al., 2018b, Lemma 4.2), the error estimate in Theorem 3.5 is optimal up to an $\varepsilon$ order.

REMARK 3.3 So far our discussion focuses on the case of a zero initial condition, i.e., $u(0)=0$. The analysis can be extended to the case of smooth initial data:

$$
\partial_{t}^{\alpha} u-\Delta u=f, \quad \text { with } u(0)=u_{0} .
$$

where $u_{0} \in D(\Delta)$ and $\partial_{t}^{\alpha} u$ denotes the Caputo fractional derivative. Then the function $w:=u-u_{0}$ satisfies (1.2) with a source $F=f+\Delta u_{0}$, for which our approach applies. The case of a nonsmooth $u_{0}$, e.g., $u_{0} \in L^{2}(\Omega)$, requires new techniques, due to a lack of regularity of the state variable $u$ (near $t=0$ ).

\section{Numerical results and discussions}

Now we present numerical experiments to illustrate the theoretical findings.

\subsection{One-dimensional examples}

We perform experiments on the unit interval $\Omega=(0,1)$. The domain $\Omega$ is divided into $M$ equally spaced subintervals with a mesh size $h=1 / M$. To discretize the fractional derivatives ${ }_{0} \partial_{t}^{\alpha} u$ and ${ }_{t} \partial_{T}^{\alpha} z$, we fix the time stepsize $\tau=T / N$. We present numerical results only for the fully discrete scheme by the Galerkin FEM in space and the L1 scheme in time, since BE-CQ gives nearly identical results.

We consider the following two examples to illustrate the analysis.

(a) $f \equiv 0$ and $u_{d}(x, t)=e^{t} x(1-x)$. 
(b) $f=(1+\cos (t)) \chi_{(1 / 2,1)}(x)$ and $u_{d}(x, t)=5 e^{t} x(1-x)$.

Throughout, the penalty parameter $\gamma$ is set to $\gamma=1$, and the lower and upper bounds $a$ and $b$ in the admissible set $U_{\text {ad }}$ to $a=0$ and $b=0.05$. The final time $T$ is fixed at $T=0.1$. The conditions from Theorems 3.3, 3.4 and 3.5 are satisfied for both examples, and thus the error estimates therein hold.

In Tables 1 and 4, we present the spatial error $e_{h}(u)$ in the $L^{\infty}\left(0, T ; L^{2}(\Omega)\right)$-norm for the semidiscrete solution $u_{h}$, defined by

$$
e_{h}(u)=\max _{1 \leqslant n \leqslant N}\left\|u_{h}\left(t_{n}\right)-u\left(t_{n}\right)\right\|_{L^{2}(\Omega)},
$$

and similarly for the approximations $z_{h}$ and $q_{h}$. The numbers in the bracket in the last column denote the theoretical rates. Since the exact solution to problem (1.2) is unavailable, we compute reference solutions on a finer mesh, i.e., the continuous solution $u\left(t_{n}\right)$ with a fixed time step $\tau=T / 1000$ and mesh size $h=1 / 1280$. The empirical rate for the spatial error $e_{h}$ is of order $O\left(h^{2}\right)$, which is consistent with the theoretical result in Theorem 3.3. For case (a), the box constraint is inactive, and thus the errors for the control $q$ and adjoint $z$ are identical (since $\gamma=1$ ).

Table 1: Spatial errors for example (a) with $N=10^{4}$.

\begin{tabular}{|c|c|cccccc|c|}
\hline$\alpha$ & $M$ & 10 & 20 & 40 & 80 & 160 & 320 & rate \\
\hline \multirow{3}{*}{0.4} & $e_{h}(u)$ & $4.57 \mathrm{e}-6$ & $1.14 \mathrm{e}-6$ & $2.86 \mathrm{e}-7$ & $7.14 \mathrm{e}-8$ & $1.79 \mathrm{e}-8$ & $4.47 \mathrm{e}-8$ & $2.00(2.00)$ \\
& $e_{h}(q)$ & $3.38 \mathrm{e}-5$ & $8.46 \mathrm{e}-6$ & $2.12 \mathrm{e}-6$ & $5.29 \mathrm{e}-7$ & $1.32 \mathrm{e}-7$ & $3.31 \mathrm{e}-8$ & $2.00(2.00)$ \\
& $e_{h}(z)$ & $3.38 \mathrm{e}-5$ & $8.46 \mathrm{e}-6$ & $2.12 \mathrm{e}-6$ & $5.29 \mathrm{e}-7$ & $1.32 \mathrm{e}-7$ & $3.31 \mathrm{e}-8$ & $2.00(2.00)$ \\
\hline \multirow{3}{*}{0.6} & $e_{h}(u)$ & $2.44 \mathrm{e}-6$ & $6.07 \mathrm{e}-7$ & $1.52 \mathrm{e}-7$ & $3.79 \mathrm{e}-8$ & $9.47 \mathrm{e}-9$ & $2.37 \mathrm{e}-9$ & $2.00(2.00)$ \\
& $e_{h}(q)$ & $3.62 \mathrm{e}-5$ & $9.04 \mathrm{e}-6$ & $2.26 \mathrm{e}-6$ & $5.65 \mathrm{e}-7$ & $1.41 \mathrm{e}-7$ & $3.53 \mathrm{e}-8$ & $2.00(2.00)$ \\
& $e_{h}(z)$ & $3.62 \mathrm{e}-5$ & $9.04 \mathrm{e}-6$ & $2.26 \mathrm{e}-6$ & $5.65 \mathrm{e}-7$ & $1.41 \mathrm{e}-7$ & $3.53 \mathrm{e}-8$ & $2.00(2.00)$ \\
\hline \multirow{3}{*}{0.8} & $e_{h}(u)$ & $8.93 \mathrm{e}-7$ & $2.21 \mathrm{e}-7$ & $5.52 \mathrm{e}-8$ & $1.40 \mathrm{e}-8$ & $3.45 \mathrm{e}-9$ & $8.62 \mathrm{e}-10$ & $2.00(2.00)$ \\
& $e_{h}(q)$ & $3.92 \mathrm{e}-5$ & $9.81 \mathrm{e}-6$ & $2.45 \mathrm{e}-6$ & $6.14 \mathrm{e}-7$ & $1.53 \mathrm{e}-7$ & $3.83 \mathrm{e}-8$ & $2.00(2.00)$ \\
& $e_{h}(z)$ & $3.92 \mathrm{e}-5$ & $9.81 \mathrm{e}-6$ & $2.45 \mathrm{e}-6$ & $6.14 \mathrm{e}-7$ & $1.53 \mathrm{e}-7$ & $3.83 \mathrm{e}-8$ & $2.00(2.00)$ \\
\hline
\end{tabular}

Table 2: Temporal errors for example (a) with $M=50$.

\begin{tabular}{|c|c|cccccc|c|}
\hline$\alpha$ & $N$ & 1000 & 2000 & 4000 & 8000 & 16000 & 32000 & rate \\
\hline \multirow{3}{*}{0.4} & $e_{\tau, 2}(u)$ & $1.70 \mathrm{e}-6$ & $9.97 \mathrm{e}-7$ & $5.77 \mathrm{e}-7$ & $3.31 \mathrm{e}-7$ & $1.88 \mathrm{e}-7$ & $1.06 \mathrm{e}-7$ & $0.83(0.90)$ \\
& $e_{\tau, 2}(q)$ & $2.02 \mathrm{e}-5$ & $1.20 \mathrm{e}-5$ & $7.06 \mathrm{e}-6$ & $4.09 \mathrm{e}-6$ & $2.34 \mathrm{e}-6$ & $1.33 \mathrm{e}-6$ & $0.82(0.90)$ \\
& $e_{\tau, 2}(z)$ & $2.02 \mathrm{e}-5$ & $1.20 \mathrm{e}-5$ & $7.06 \mathrm{e}-6$ & $4.09 \mathrm{e}-6$ & $2.34 \mathrm{e}-6$ & $1.33 \mathrm{e}-6$ & $0.82(0.90)$ \\
\hline \multirow{3}{*}{0.6} & $e_{\tau, 2}(u)$ & $6.58 \mathrm{e}-7$ & $3.47 \mathrm{e}-7$ & $1.82 \mathrm{e}-7$ & $9.47 \mathrm{e}-8$ & $4.90 \mathrm{e}-8$ & $2.53 \mathrm{e}-8$ & $0.96(1.00)$ \\
& $e_{\tau, 2}(q)$ & $8.25 \mathrm{e}-6$ & $4.37 \mathrm{e}-6$ & $2.29 \mathrm{e}-6$ & $1.20 \mathrm{e}-6$ & $6.22 \mathrm{e}-7$ & $3.21 \mathrm{e}-7$ & $0.95(1.00)$ \\
& $e_{\tau, 2}(z)$ & $8.25 \mathrm{e}-6$ & $4.37 \mathrm{e}-6$ & $2.29 \mathrm{e}-6$ & $1.20 \mathrm{e}-6$ & $6.22 \mathrm{e}-7$ & $3.21 \mathrm{e}-7$ & $0.95(1.00)$ \\
\hline \multirow{3}{*}{0.8} & $e_{\tau, 2}(u)$ & $2.68 \mathrm{e}-7$ & $1.38 \mathrm{e}-7$ & $7.07 \mathrm{e}-8$ & $3.62 \mathrm{e}-8$ & $1.84 \mathrm{e}-8$ & $9.38 \mathrm{e}-9$ & $0.97(1.00)$ \\
& $e_{\tau, 2}(q)$ & $3.80 \mathrm{e}-6$ & $1.95 \mathrm{e}-6$ & $1.00 \mathrm{e}-6$ & $5.12 \mathrm{e}-7$ & $2.61 \mathrm{e}-7$ & $1.33 \mathrm{e}-7$ & $0.98(1.00)$ \\
& $e_{\tau, 2}(z)$ & $3.80 \mathrm{e}-6$ & $1.95 \mathrm{e}-6$ & $1.00 \mathrm{e}-6$ & $5.12 \mathrm{e}-7$ & $2.61 \mathrm{e}-7$ & $1.33 \mathrm{e}-7$ & $0.98(1.00)$ \\
\hline
\end{tabular}

Next, to examine the convergence in time, we compute the $\ell^{2}\left(L^{2}(\Omega)\right)$ and $\ell^{\infty}\left(L^{2}(\Omega)\right)$ temporal errors $e_{\tau, 2}(u)$ and $e_{\tau, \infty}(u)$ for the fully discrete solutions $U_{h}^{n}$, respectively, defined by

$$
e_{\tau, 2}(u)=\left\|\left(U_{h}^{n}-u_{h}\left(t_{n}\right)\right)_{n=1}^{N}\right\|_{\ell^{2}\left(L^{2}(\Omega)\right)} \quad \text { and } \quad e_{\tau, \infty}(u)=\left\|\left(U_{h}^{n}-u_{h}\left(t_{n}\right)\right)_{n=1}^{N}\right\|_{\ell^{\infty}\left(L^{2}(\Omega)\right)},
$$

and similarly for the approximations $Z_{h}^{n}$ and $Q_{h}^{n}$. The reference semidiscrete solutions are computed with $h=1 / 50$ and $\tau=1 /\left(64 \times 10^{4}\right)$. Numerical experiments show that the empirical rate for the temporal discretization error is of order $O\left(\tau^{\min \left(\frac{1}{2}+\alpha, 1\right)}\right)$ and $O\left(\tau^{\alpha}\right)$ in the $\ell^{2}\left(L^{2}(\Omega)\right)$ and $\ell^{\infty}\left(L^{2}(\Omega)\right)$ norms, respectively, cf. Tables $2-3$ and 5-6, for cases (a) and (b). These results agree well with the theoretical predictions from Theorems 3.4 and 3.5, and thus fully support the error analysis in Section 3. In Fig. 1, we plot the optimal control $q$, the state $u$ and the adjoint $z$. One clearly observes the 
weak solution singularity at $t=0$ for the state $u$ and at $t=T$ for the adjoint $z$. The latter is especially pronounced for case (b). The weak solution singularity is due to the incompatibility of the source term with the zero initial/terminal data.

Table 3: Pointwise-in-time temporal errors for example (a) with $M=50$.

\begin{tabular}{|c|c|cccccc|c|}
\hline$\alpha$ & $N$ & 1000 & 2000 & 4000 & 8000 & 16000 & 32000 & rate \\
\hline \multirow{3}{*}{0.4} & $e_{\tau, \infty}(u)$ & $3.47 \mathrm{e}-5$ & $2.78 \mathrm{e}-5$ & $2.20 \mathrm{e}-5$ & $1.72 \mathrm{e}-5$ & $1.33 \mathrm{e}-5$ & $1.03 \mathrm{e}-5$ & $0.37(0.40)$ \\
& $e_{\tau, \infty}(q)$ & $4.47 \mathrm{e}-4$ & $3.58 \mathrm{e}-4$ & $2.83 \mathrm{e}-4$ & $2.21 \mathrm{e}-4$ & $1.72 \mathrm{e}-4$ & $1.33 \mathrm{e}-4$ & $0.37(0.40)$ \\
& $e_{\tau, \infty}(z)$ & $4.47 \mathrm{e}-4$ & $3.58 \mathrm{e}-4$ & $2.83 \mathrm{e}-4$ & $2.21 \mathrm{e}-4$ & $1.72 \mathrm{e}-4$ & $1.33 \mathrm{e}-4$ & $0.37(0.40)$ \\
\hline \multirow{3}{*}{0.6} & $e_{\tau, \infty}(u)$ & $5.72 \mathrm{e}-6$ & $3.79 \mathrm{e}-6$ & $2.51 \mathrm{e}-6$ & $1.66 \mathrm{e}-6$ & $1.09 \mathrm{e}-6$ & $7.22 \mathrm{e}-7$ & $0.60(0.60)$ \\
& $e_{\tau, \infty}(q)$ & $7.64 \mathrm{e}-5$ & $5.06 \mathrm{e}-5$ & $3.35 \mathrm{e}-5$ & $2.21 \mathrm{e}-5$ & $1.46 \mathrm{e}-5$ & $9.64 \mathrm{e}-6$ & $0.60(0.60)$ \\
& $e_{\tau, \infty}(z)$ & $7.64 \mathrm{e}-5$ & $5.06 \mathrm{e}-5$ & $3.35 \mathrm{e}-5$ & $2.21 \mathrm{e}-5$ & $1.46 \mathrm{e}-5$ & $9.64 \mathrm{e}-6$ & $0.60(0.60)$ \\
\hline \multirow{3}{*}{0.8} & $e_{\tau, \infty}(u)$ & $6.93 \mathrm{e}-7$ & $3.97 \mathrm{e}-7$ & $2.28 \mathrm{e}-7$ & $1.31 \mathrm{e}-7$ & $7.50 \mathrm{e}-8$ & $4.31 \mathrm{e}-8$ & $0.80(0.80)$ \\
& $e_{\tau, \infty}(q)$ & $9.85 \mathrm{e}-6$ & $5.65 \mathrm{e}-6$ & $3.24 \mathrm{e}-6$ & $1.86 \mathrm{e}-6$ & $1.07 \mathrm{e}-6$ & $6.13 \mathrm{e}-7$ & $0.80(0.80)$ \\
& $e_{\tau, \infty}(z)$ & $9.85 \mathrm{e}-6$ & $5.65 \mathrm{e}-6$ & $3.24 \mathrm{e}-6$ & $1.86 \mathrm{e}-6$ & $1.07 \mathrm{e}-6$ & $6.13 \mathrm{e}-7$ & $0.80(0.80)$ \\
\hline
\end{tabular}
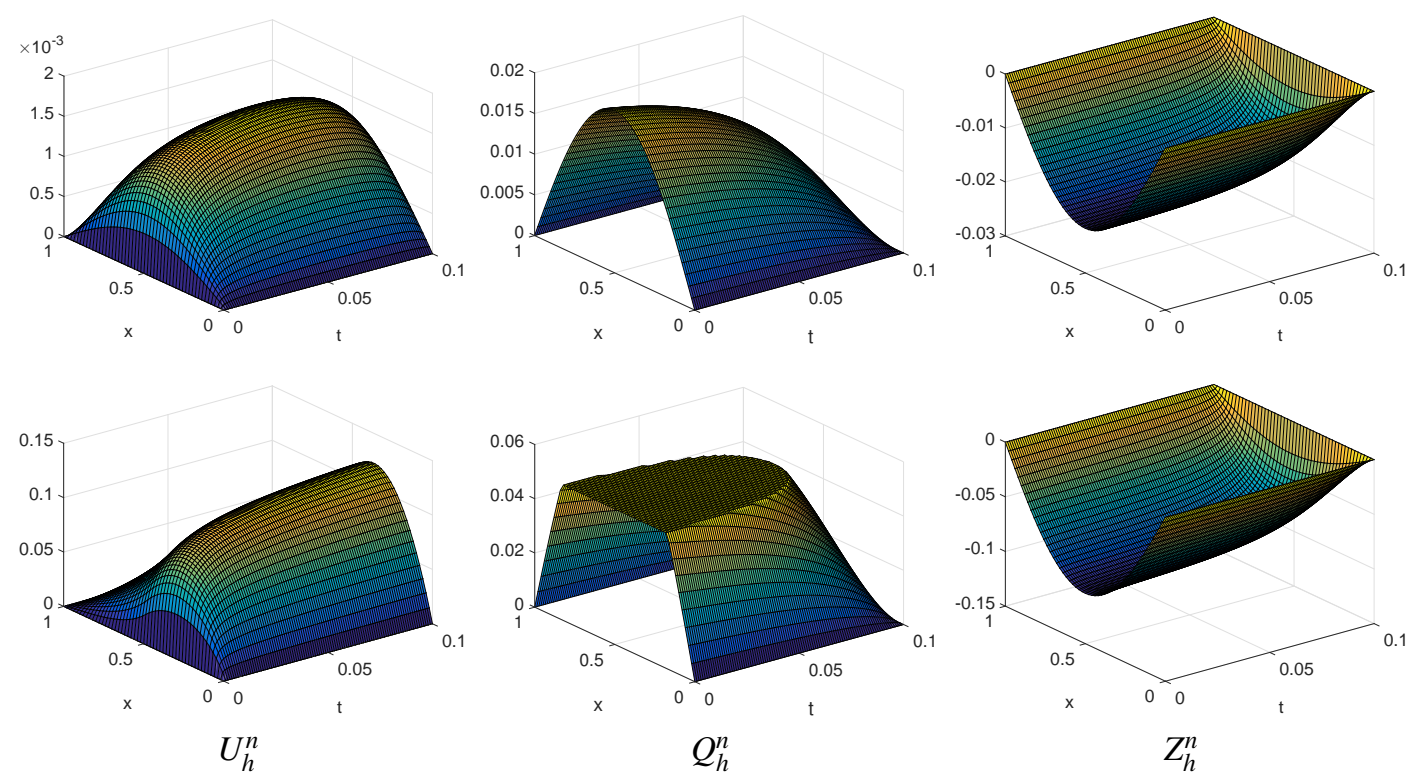

FIG. 1: Plot of $U_{h}^{n}, Q_{h}^{n}$ and $Z_{h}^{n}$ for example (a) (top) and (b) (bottom).

\subsection{Two-dimensional example}

Now, we present numerical results of a two-dimensional example. The domain $\Omega$ is taken to be the unit square $\Omega=(0,1)^{2}$. To discretize the problem, we first divide the unit interval $(0,1)$ into $M$ equally spaced subintervals so that $\Omega$ is divided into $M^{2}$ small squares, and then obtain a uniform triangulation for the domain $\Omega$ by connecting the diagonal of each small square. We consider the following data:

(c) $f \equiv 0, u_{d}(x, t)=5 e^{t} x(1-x) y^{2} \sin (2 \pi y)$ and $u(x, 0)=x(1-x) y(1-y)$.

In the experiment, we set an active admissible set $U_{\text {ad }}$ with lower bound $a=-0.01$ and upper bound $b=0.01$. We take $T=0.01$ and $\gamma=1$, for evaluating both the spatial and temporal errors (i.e., $e_{h}, e_{\tau, 2}$ and $e_{\tau, \infty}$. Note that in this example, the initial data $v$ is nonzero, but it is smooth and compatible with 
Table 4: Spatial errors for example (b) with $N=10^{4}$.

\begin{tabular}{|c|c|cccccc|c|}
\hline$\alpha$ & $N$ & 10 & 20 & 40 & 80 & 160 & 320 & rate \\
\hline \multirow{3}{*}{0.4} & $e_{h}(u)$ & $1.86 \mathrm{e}-4$ & $4.72 \mathrm{e}-5$ & $1.16 \mathrm{e}-5$ & $2.82 \mathrm{e}-6$ & $7.39 \mathrm{e}-7$ & $1.84 \mathrm{e}-7$ & $2.00(2.00)$ \\
& $e_{h}(q)$ & $1.59 \mathrm{e}-4$ & $3.97 \mathrm{e}-4$ & $9.92 \mathrm{e}-6$ & $2.48 \mathrm{e}-6$ & $6.19 \mathrm{e}-7$ & $1.55 \mathrm{e}-7$ & $2.00(2.00)$ \\
& $e_{h}(z)$ & $1.78 \mathrm{e}-4$ & $4.44 \mathrm{e}-5$ & $1.11 \mathrm{e}-5$ & $2.78 \mathrm{e}-6$ & $6.94 \mathrm{e}-7$ & $1.74 \mathrm{e}-7$ & $2.00(2.00)$ \\
\hline \multirow{3}{*}{0.6} & $e_{h}(u)$ & $1.99 \mathrm{e}-4$ & $4.93 \mathrm{e}-5$ & $1.20 \mathrm{e}-5$ & $3.14 \mathrm{e}-6$ & $7.83 \mathrm{e}-7$ & $1.94 \mathrm{e}-7$ & $2.00(2.00)$ \\
& $e_{h}(q)$ & $1.66 \mathrm{e}-4$ & $4.15 \mathrm{e}-5$ & $1.04 \mathrm{e}-5$ & $2.60 \mathrm{e}-6$ & $6.50 \mathrm{e}-7$ & $1.63 \mathrm{e}-7$ & $2.00(2.00)$ \\
& $e_{h}(z)$ & $1.86 \mathrm{e}-4$ & $4.66 \mathrm{e}-5$ & $1.16 \mathrm{e}-5$ & $2.91 \mathrm{e}-6$ & $7.28 \mathrm{e}-7$ & $1.82 \mathrm{e}-7$ & $2.00(2.00)$ \\
\hline \multirow{3}{*}{0.8} & $e_{h}(u)$ & $2.19 \mathrm{e}-4$ & $5.31 \mathrm{e}-5$ & $1.35 \mathrm{e}-5$ & $3.35 \mathrm{e}-6$ & $8.39 \mathrm{e}-7$ & $2.10 \mathrm{e}-7$ & $2.00(2.00)$ \\
& $e_{h}(q)$ & $1.71 \mathrm{e}-4$ & $4.29 \mathrm{e}-5$ & $1.07 \mathrm{e}-5$ & $2.68 \mathrm{e}-6$ & $6.70 \mathrm{e}-7$ & $1.68 \mathrm{e}-7$ & $2.00(2.00)$ \\
& $e_{h}(z)$ & $1.96 \mathrm{e}-4$ & $4.91 \mathrm{e}-5$ & $1.23 \mathrm{e}-5$ & $3.07 \mathrm{e}-6$ & $7.66 \mathrm{e}-7$ & $1.92 \mathrm{e}-7$ & $2.00(2.00)$ \\
\hline
\end{tabular}

Table 5: Temporal errors for example (b) with $M=50$.

\begin{tabular}{|c|c|cccccc|c|}
\hline$\alpha$ & $N$ & 1000 & 2000 & 4000 & 8000 & 16000 & 32000 & rate \\
\hline \multirow{3}{*}{0.4} & $e_{\tau, 2}(u)$ & $1.05 \mathrm{e}-4$ & $6.34 \mathrm{e}-5$ & $3.79 \mathrm{e}-5$ & $2.24 \mathrm{e}-5$ & $1.31 \mathrm{e}-5$ & $7.59 \mathrm{e}-6$ & $0.79(0.90)$ \\
& $e_{\tau, 2}(q)$ & $9.00 \mathrm{e}-5$ & $5.43 \mathrm{e}-5$ & $3.21 \mathrm{e}-5$ & $1.87 \mathrm{e}-5$ & $1.07 \mathrm{e}-5$ & $6.10 \mathrm{e}-6$ & $0.81(0.90)$ \\
& $e_{\tau, 2}(z)$ & $9.36 \mathrm{e}-5$ & $5.57 \mathrm{e}-5$ & $3.26 \mathrm{e}-5$ & $1.89 \mathrm{e}-5$ & $1.08 \mathrm{e}-5$ & $6.15 \mathrm{e}-6$ & $0.82(0.90)$ \\
\hline \multirow{3}{*}{0.6} & $e_{\tau, 2}(u)$ & $4.67 \mathrm{e}-5$ & $2.50 \mathrm{e}-5$ & $1.33 \mathrm{e}-5$ & $6.99 \mathrm{e}-6$ & $3.65 \mathrm{e}-6$ & $1.90 \mathrm{e}-6$ & $0.94(1.00)$ \\
& $e_{\tau, 2}(q)$ & $3.66 \mathrm{e}-5$ & $1.95 \mathrm{e}-5$ & $1.03 \mathrm{e}-5$ & $5.40 \mathrm{e}-6$ & $2.81 \mathrm{e}-6$ & $1.45 \mathrm{e}-6$ & $0.95(1.00)$ \\
& $e_{\tau, 2}(z)$ & $3.83 \mathrm{e}-5$ & $2.03 \mathrm{e}-5$ & $1.07 \mathrm{e}-5$ & $5.56 \mathrm{e}-6$ & $2.89 \mathrm{e}-6$ & $1.49 \mathrm{e}-6$ & $0.95(1.00)$ \\
\hline \multirow{3}{*}{0.8} & $e_{\tau, 2}(u)$ & $2.23 \mathrm{e}-5$ & $1.14 \mathrm{e}-5$ & $5.85 \mathrm{e}-6$ & $2.99 \mathrm{e}-6$ & $1.52 \mathrm{e}-6$ & $7.74 \mathrm{e}-7$ & $0.98(1.00)$ \\
& $e_{\tau, 2}(q)$ & $1.58 \mathrm{e}-5$ & $8.23 \mathrm{e}-6$ & $4.25 \mathrm{e}-6$ & $2.19 \mathrm{e}-6$ & $1.12 \mathrm{e}-6$ & $5.73 \mathrm{e}-7$ & $0.97(1.00)$ \\
& $e_{\tau, 2}(z)$ & $1.78 \mathrm{e}-5$ & $9.17 \mathrm{e}-6$ & $4.70 \mathrm{e}-6$ & $2.40 \mathrm{e}-6$ & $1.22 \mathrm{e}-6$ & $6.23 \mathrm{e}-7$ & $0.98(1.00)$ \\
\hline
\end{tabular}

Table 6: Pointwise-in-time temporal errors for example (b) with $M=50$.

\begin{tabular}{|c|c|cccccc|c|}
\hline$\alpha$ & $N$ & 1000 & 2000 & 4000 & 8000 & 16000 & 32000 & rate \\
\hline \multirow{3}{*}{0.4} & $e_{\tau, \infty}(u)$ & $2.40 \mathrm{e}-3$ & $1.98 \mathrm{e}-3$ & $1.62 \mathrm{e}-3$ & $1.31 \mathrm{e}-3$ & $1.04 \mathrm{e}-3$ & $8.25 \mathrm{e}-4$ & $0.34(0.40)$ \\
& $e_{\tau, \infty}(q)$ & $2.07 \mathrm{e}-3$ & $1.65 \mathrm{e}-3$ & $1.31 \mathrm{e}-3$ & $1.02 \mathrm{e}-3$ & $7.95 \mathrm{e}-4$ & $6.14 \mathrm{e}-4$ & $0.37(0.40)$ \\
& $e_{\tau, \infty}(z)$ & $2.07 \mathrm{e}-3$ & $1.65 \mathrm{e}-3$ & $1.31 \mathrm{e}-3$ & $1.02 \mathrm{e}-3$ & $7.95 \mathrm{e}-4$ & $6.14 \mathrm{e}-4$ & $0.37(0.40)$ \\
\hline \multirow{3}{*}{0.6} & $e_{\tau, \infty}(u)$ & $5.11 \mathrm{e}-4$ & $3.45 \mathrm{e}-4$ & $2.32 \mathrm{e}-4$ & $1.55 \mathrm{e}-4$ & $1.03 \mathrm{e}-4$ & $6.85 \mathrm{e}-5$ & $0.59(0.60)$ \\
& $e_{\tau, \infty}(q)$ & $3.54 \mathrm{e}-4$ & $2.35 \mathrm{e}-4$ & $1.55 \mathrm{e}-4$ & $1.03 \mathrm{e}-4$ & $6.77 \mathrm{e}-5$ & $4.47 \mathrm{e}-5$ & $0.60(0.60)$ \\
& $e_{\tau, \infty}(z)$ & $3.54 \mathrm{e}-4$ & $2.35 \mathrm{e}-4$ & $1.55 \mathrm{e}-4$ & $1.03 \mathrm{e}-4$ & $6.77 \mathrm{e}-5$ & $4.47 \mathrm{e}-5$ & $0.60(0.60)$ \\
\hline \multirow{3}{*}{0.8} & $e_{\tau, \infty}(u)$ & $6.96 \mathrm{e}-5$ & $4.03 \mathrm{e}-5$ & $2.33 \mathrm{e}-5$ & $1.34 \mathrm{e}-5$ & $7.68 \mathrm{e}-6$ & $4.41 \mathrm{e}-6$ & $0.80(0.80)$ \\
& $e_{\tau, \infty}(q)$ & $4.60 \mathrm{e}-5$ & $2.63 \mathrm{e}-5$ & $1.51 \mathrm{e}-5$ & $8.67 \mathrm{e}-6$ & $4.98 \mathrm{e}-6$ & $2.86 \mathrm{e}-6$ & $0.80(0.80)$ \\
& $e_{\tau, \infty}(z)$ & $4.60 \mathrm{e}-5$ & $2.63 \mathrm{e}-5$ & $1.51 \mathrm{e}-5$ & $8.67 \mathrm{e}-6$ & $4.98 \mathrm{e}-6$ & $2.86 \mathrm{e}-6$ & $0.80(0.80)$ \\
\hline
\end{tabular}

the zero Dirichlet boundary condition. Thus, one may reformulate the control problem according to Remark 3.3, and the analysis still applies. The numerical results are given in Tables 7, 8 and 9, which indicate that the empirical convergence rates for both spatial and temporal errors agree well with the theoretical ones in Theorems 3.3, 3.4 and 3.5.

\section{Conclusions}

In this work, we have developed a complete numerical analysis of a fully discrete scheme for a distributed optimal control problem governed by a subdiffusion equation, with box constraint on the control variable, and derived nearly sharp pointwise-in-time error estimates for both space and time discretizations. These estimates agree well with the empirical rates observed in the numerical experiments. The theoretical and numerical results show the adverse influence of the fractional derivatives on the convergence rate when the fractional order $\alpha$ is small. 
Table 7: Spatial errors for example (c) with $N=500$.

\begin{tabular}{|c|c|ccccc|c|}
\hline$\alpha$ & $M$ & 5 & 10 & 20 & 40 & 80 & rate \\
\hline \multirow{3}{*}{0.4} & $e_{h}(u)$ & $1.67 \mathrm{e}-3$ & $4.31 \mathrm{e}-4$ & $1.08 \mathrm{e}-4$ & $2.73 \mathrm{e}-5$ & $6.91 \mathrm{e}-6$ & $1.99(2.00)$ \\
& $e_{h}(q)$ & $6.11 \mathrm{e}-4$ & $1.48 \mathrm{e}-4$ & $3.75 \mathrm{e}-5$ & $9.44 \mathrm{e}-6$ & $2.38 \mathrm{e}-6$ & $1.98(2.00)$ \\
& $e_{h}(z)$ & $1.92 \mathrm{e}-3$ & $5.08 \mathrm{e}-4$ & $1.29 \mathrm{e}-4$ & $3.25 \mathrm{e}-5$ & $8.22 \mathrm{e}-6$ & $1.98(2.00)$ \\
\hline \multirow{3}{*}{0.6} & $e_{h}(u)$ & $1.82 \mathrm{e}-3$ & $4.64 \mathrm{e}-4$ & $1.17 \mathrm{e}-4$ & $2.94 \mathrm{e}-5$ & $7.51 \mathrm{e}-6$ & $1.98(2.00)$ \\
& $e_{h}(q)$ & $5.51 \mathrm{e}-4$ & $1.34 \mathrm{e}-4$ & $3.28 \mathrm{e}-5$ & $8.37 \mathrm{e}-6$ & $2.12 \mathrm{e}-6$ & $1.98(2.00)$ \\
& $e_{h}(z)$ & $1.98 \mathrm{e}-3$ & $5.22 \mathrm{e}-4$ & $1.33 \mathrm{e}-4$ & $3.34 \mathrm{e}-5$ & $8.44 \mathrm{e}-6$ & $1.98(2.00)$ \\
\hline \multirow{3}{*}{0.8} & $e_{h}(u)$ & $1.85 \mathrm{e}-3$ & $4.68 \mathrm{e}-4$ & $1.18 \mathrm{e}-4$ & $2.97 \mathrm{e}-5$ & $7.61 \mathrm{e}-6$ & $1.97(2.00)$ \\
& $e_{h}(q)$ & $4.51 \mathrm{e}-4$ & $1.21 \mathrm{e}-4$ & $2.68 \mathrm{e}-5$ & $7.13 \mathrm{e}-6$ & $1.80 \mathrm{e}-6$ & $1.98(2.00)$ \\
& $e_{h}(z)$ & $2.02 \mathrm{e}-3$ & $5.34 \mathrm{e}-4$ & $1.35 \mathrm{e}-4$ & $3.41 \mathrm{e}-5$ & $8.62 \mathrm{e}-6$ & $1.98(2.00)$ \\
\hline
\end{tabular}

Table 8: Temporal errors for example (c) with $M=50$.

\begin{tabular}{|c|c|cccccc|c|}
\hline$\alpha$ & $N$ & 100 & 200 & 400 & 800 & 1600 & 3200 & rate \\
\hline \multirow{3}{*}{0.4} & $e_{\tau, 2}(u)$ & $3.83 \mathrm{e}-5$ & $2.38 \mathrm{e}-5$ & $1.46 \mathrm{e}-5$ & $8.74 \mathrm{e}-6$ & $5.16 \mathrm{e}-6$ & $3.01 \mathrm{e}-6$ & $0.78(0.90)$ \\
& $e_{\tau, 2}(q)$ & $3.14 \mathrm{e}-5$ & $1.80 \mathrm{e}-5$ & $1.04 \mathrm{e}-5$ & $6.03 \mathrm{e}-6$ & $3.54 \mathrm{e}-6$ & $2.08 \mathrm{e}-6$ & $0.77(0.90)$ \\
& $e_{\tau, 2}(z)$ & $3.14 \mathrm{e}-5$ & $1.96 \mathrm{e}-5$ & $1.20 \mathrm{e}-5$ & $7.23 \mathrm{e}-6$ & $4.29 \mathrm{e}-6$ & $2.51 \mathrm{e}-6$ & $0.78(0.90)$ \\
\hline \multirow{3}{*}{0.6} & $e_{\tau, 2}(u)$ & $1.76 \mathrm{e}-5$ & $9.49 \mathrm{e}-6$ & $5.06 \mathrm{e}-6$ & $2.68 \mathrm{e}-6$ & $1.40 \mathrm{e}-6$ & $7.32 \mathrm{e}-7$ & $0.94(1.00)$ \\
& $e_{\tau, 2}(q)$ & $1.34 \mathrm{e}-5$ & $7.19 \mathrm{e}-6$ & $3.82 \mathrm{e}-6$ & $2.02 \mathrm{e}-6$ & $1.06 \mathrm{e}-6$ & $5.53 \mathrm{e}-7$ & $0.94(1.00)$ \\
& $e_{\tau, 2}(z)$ & $1.44 \mathrm{e}-5$ & $7.81 \mathrm{e}-6$ & $4.17 \mathrm{e}-6$ & $2.21 \mathrm{e}-6$ & $1.16 \mathrm{e}-6$ & $6.06 \mathrm{e}-7$ & $0.94(1.00)$ \\
\hline \multirow{3}{*}{0.8} & $e_{\tau, 2}(u)$ & $6.49 \mathrm{e}-6$ & $3.41 \mathrm{e}-6$ & $1.78 \mathrm{e}-6$ & $9.22 \mathrm{e}-7$ & $4.75 \mathrm{e}-7$ & $2.44 \mathrm{e}-7$ & $0.96(1.00)$ \\
& $e_{\tau, 2}(q)$ & $4.85 \mathrm{e}-6$ & $2.53 \mathrm{e}-6$ & $1.31 \mathrm{e}-6$ & $6.72 \mathrm{e}-7$ & $3.44 \mathrm{e}-7$ & $1.76 \mathrm{e}-7$ & $0.97(1.00)$ \\
& $e_{\tau, 2}(z)$ & $5.27 \mathrm{e}-6$ & $2.77 \mathrm{e}-6$ & $1.44 \mathrm{e}-6$ & $7.49 \mathrm{e}-7$ & $3.86 \mathrm{e}-7$ & $1.98 \mathrm{e}-7$ & $0.96(1.00)$ \\
\hline
\end{tabular}

Table 9: Pointwise-in-time temporal errors for example (c) with $M=50$.

\begin{tabular}{|c|c|cccccc|c|}
\hline$\alpha$ & $N$ & 100 & 200 & 400 & 800 & 1600 & 3200 & rate \\
\hline \multirow{3}{*}{0.4} & $e_{\tau, \infty}(u)$ & $3.00 \mathrm{e}-4$ & $2.53 \mathrm{e}-4$ & $2.08 \mathrm{e}-4$ & $1.68 \mathrm{e}-4$ & $1.34 \mathrm{e}-4$ & $1.06 \mathrm{e}-4$ & $0.34(0.40)$ \\
& $e_{\tau, \infty}(q)$ & $1.95 \mathrm{e}-4$ & $1.62 \mathrm{e}-4$ & $1.36 \mathrm{e}-4$ & $1.13 \mathrm{e}-4$ & $9.35 \mathrm{e}-5$ & $7.72 \mathrm{e}-5$ & $0.28(0.40)$ \\
& $e_{\tau, \infty}(z)$ & $2.47 \mathrm{e}-4$ & $2.09 \mathrm{e}-4$ & $1.73 \mathrm{e}-4$ & $1.41 \mathrm{e}-4$ & $1.13 \mathrm{e}-4$ & $8.92 \mathrm{e}-5$ & $0.34(0.40)$ \\
\hline \multirow{3}{*}{0.6} & $e_{\tau, \infty}(u)$ & $6.47 \mathrm{e}-5$ & $4.33 \mathrm{e}-5$ & $2.89 \mathrm{e}-5$ & $1.92 \mathrm{e}-5$ & $1.27 \mathrm{e}-5$ & $8.44 \mathrm{e}-6$ & $0.59(0.60)$ \\
& $e_{\tau, \infty}(q)$ & $3.65 \mathrm{e}-5$ & $3.91 \mathrm{e}-5$ & $2.62 \mathrm{e}-5$ & $1.75 \mathrm{e}-5$ & $1.17 \mathrm{e}-5$ & $7.85 \mathrm{e}-6$ & $0.58(0.60)$ \\
& $e_{\tau, \infty}(z)$ & $5.45 \mathrm{e}-5$ & $3.66 \mathrm{e}-5$ & $2.44 \mathrm{e}-5$ & $1.63 \mathrm{e}-5$ & $1.08 \mathrm{e}-5$ & $7.17 \mathrm{e}-5$ & $0.59(0.60)$ \\
\hline \multirow{3}{*}{0.8} & $e_{\tau, \infty}(u)$ & $8.64 \mathrm{e}-6$ & $4.96 \mathrm{e}-6$ & $2.86 \mathrm{e}-6$ & $1.64 \mathrm{e}-6$ & $9.44 \mathrm{e}-7$ & $5.42 \mathrm{e}-7$ & $0.80(0.80)$ \\
& $e_{\tau, \infty}(q)$ & $8.70 \mathrm{e}-6$ & $4.99 \mathrm{e}-6$ & $2.84 \mathrm{e}-6$ & $1.63 \mathrm{e}-6$ & $9.24 \mathrm{e}-7$ & $5.25 \mathrm{e}-7$ & $0.82(0.80)$ \\
& $e_{\tau, \infty}(z)$ & $7.23 \mathrm{e}-6$ & $4.16 \mathrm{e}-6$ & $2.39 \mathrm{e}-6$ & $1.38 \mathrm{e}-6$ & $7.92 \mathrm{e}-7$ & $4.55 \mathrm{e}-7$ & $0.80(0.80)$ \\
\hline
\end{tabular}

\section{A. Proof of Lemma 2.4}

Proof. By Sobolev embedding, $W^{s, p}\left(0,1 ; L^{2}(\Omega)\right) \hookrightarrow C\left([0,1] ; L^{2}(\Omega)\right)$ for $s \in(1 / p, 1]$, and thus we can define an interpolation operator $\Pi$ by $\Pi v(\hat{t})=v(1)$, for $\hat{t} \in(0,1)$, for any $v \in W^{s, p}\left(0,1 ; L^{2}(\Omega)\right)$. The operator $E=I-\Pi$ is bounded from $W^{s, p}\left(0,1 ; L^{2}(\Omega)\right)$ to $L^{2}\left(0,1 ; L^{2}(\Omega)\right)$ :

$$
\|E v\|_{L^{2}\left(0,1 ; L^{2}(\Omega)\right)}=\|(I-\Pi) v\|_{L^{2}\left(0,1 ; L^{2}(\Omega)\right)} \leqslant c\|v\|_{W^{s, p}\left(0,1 ; L^{2}(\Omega)\right)} .
$$

By the fractional Poincaré inequality (cf. Hurri-Syrjänen \& Vähäkangas (2013)), we have

$$
\begin{aligned}
\|E v\|_{L^{p}\left(0,1 ; L^{2}(\Omega)\right)} & =\inf _{p \in \mathbb{R}}\|E(v-p)\|_{L^{p}\left(0,1 ; L^{2}(\Omega)\right)} \leqslant c \inf _{p \in \mathbb{R}}\|v-p\|_{W^{s, p}\left(0,1 ; L^{2}(\Omega)\right)} \\
& \leqslant c|v|_{W^{s, p}\left(0,1 ; L^{2}(\Omega)\right)},
\end{aligned}
$$


where the seminorm $|\cdot|_{W^{s, p}\left(0, T ; L^{2}(\Omega)\right)}$ is defined in (2.4). By Hölder's inequality, we obtain

$$
\begin{aligned}
& \left\|\left(v\left(t_{n}\right)-\bar{v}^{n}\right)_{n=1}^{N}\right\|_{\ell p\left(L^{2}(\Omega)\right)}^{p}=\tau \sum_{n=1}^{N}\left\|v\left(t_{n}\right)-\tau^{-1} \int_{t_{n-1}}^{t_{n}} v(t) \mathrm{d} t\right\|_{L^{2}(\Omega)}^{p} \\
= & \tau^{1-p} \sum_{n=1}^{N}\left\|\int_{t_{n-1}}^{t_{n}}\left(v\left(t_{n}\right)-v(t)\right) \mathrm{d} t\right\|_{L^{2}(\Omega)}^{p} \leqslant \sum_{n=1}^{N} \int_{t_{n-1}}^{t_{n}}\left\|v\left(t_{n}\right)-v(t)\right\|_{L^{2}(\Omega)}^{p} \mathrm{~d} t .
\end{aligned}
$$

Let $\widehat{v}_{n}(\hat{t})=v\left(t_{n-1}+\tau \hat{t}\right)$, for $\hat{t} \in[0,1], n=1, \ldots, N$. Then $\widehat{v}_{n}(\hat{t}) \in W^{s, p}\left(0,1 ; L^{2}(\Omega)\right)$ and by (A.1),

$$
\begin{aligned}
\left\|\left(v\left(t_{n}\right)-\bar{v}^{n}\right)_{n=1}^{N}\right\|_{\ell p\left(L^{2}(\Omega)\right)}^{p} & \leqslant \tau \sum_{n=1}^{N} \int_{0}^{1}\left\|\Pi \widehat{v}_{n}-\widehat{v}_{n}\right\|_{L^{2}(\Omega)}^{p}(\hat{t}) \mathrm{d} \hat{t} \leqslant c \tau \sum_{n=1}^{N}\left|\widehat{v}_{n}\right|_{W^{s, p}\left(0,1 ; L^{2}(\Omega)\right)}^{p} \\
& \leqslant c \tau \sum_{n=1}^{N} \int_{0}^{1} \int_{0}^{1} \frac{\left\|\widehat{v}_{n}(\hat{t})-\widehat{v}_{n}(\hat{\xi})\right\|_{L^{2}(\Omega)}^{p} \mathrm{~d} \hat{t} \mathrm{~d} \hat{\xi}}{|\hat{t}-\hat{\xi}|^{1+p s}} \\
& =c \tau^{p s} \sum_{n=1}^{N} \int_{t_{n-1}}^{t_{n}} \int_{t_{n-1}}^{t_{n}} \frac{\|v(t)-v(\xi)\|_{L^{2}(\Omega)}^{p}}{|t-\xi|^{1+p s}} \mathrm{~d} t \boldsymbol{d} \\
& \leqslant c \tau^{p s} \int_{0}^{T} \int_{0}^{T} \frac{\|v(t)-v(\xi)\|_{L^{2}(\Omega)}^{p}}{|t-\xi|^{1+p s}} \mathrm{~d} \mathrm{~d}=c \tau^{p s}|v|_{W^{s, p}\left(0, T ; L^{2}(\Omega)\right)}^{p},
\end{aligned}
$$

which implies the desired assertion.

\section{REFERENCES}

Adams, E. E. \& Gelhar, L. W. (1992) Field study of dispersion in a heterogeneous aquifer: 2. spatial moments analysis. Water Res. Research, 28, 3293-3307.

Akrivis, G., LI, B. \& LUBICH, C. (2017) Combining maximal regularity and energy estimates for time discretizations of quasilinear parabolic equations. Math. Comp., 86, 1527-1552.

Antil, H., OtÁRola, E. \& SAlgado, A. J. (2016) A space-time fractional optimal control problem: analysis and discretization. SIAM J. Control Optim., 54, 1295-1328.

Arendt, W., Batty, C. J., Hieber, M. \& Neubrander, F. (2011) Vector-valued Laplace Transforms and Cauchy Problems, Second Edition, second edn. Birkhäuser.

Bajlekova, E. G. (2001) Fractional Evolution Equations in Banach Spaces. Ph.D. thesis, Eindhoven University of Technology.

Bergh, J. \& LofSTrom, J. (2012) Interpolation Spaces: an Introduction. Berlin: Springer.

Chrysafinos, K. \& KARATZAS, E. N. (2014) Error estimates for discontinuous Galerkin time-stepping schemes for Robin boundary control problems constrained to parabolic PDEs. SIAM J. Numer. Anal., 52, 2837-2862.

DU, N., WANG, H. \& LIU, W. (2016) A fast gradient projection method for a constrained fractional optimal control. J. Sci. Comput., 68, 1-20.

Fujishiro, K. \& Yамамото, M. (2014) Approximate controllability for fractional diffusion equations by interior control. Appl. Anal., 93, 1793-1810.

Gong, W., HinZe, M. \& ZHOU, Z. (2014) A priori error analysis for finite element approximation of parabolic optimal control problems with pointwise control. SIAM J. Control Optim., 52, 97-119.

Gorenflo, R., Luchкo, Y. \& Yамамото, M. (2015) Time-fractional diffusion equation in the fractional Sobolev spaces. Fract. Calc. Appl. Anal., 18, 799-820.

HATANO, Y. \& HATANO, N. (1998) Dispersive transport of ions in column experiments: An explanation of long-tailed profiles. Water Res. Research, 34, 1027-1033.

HinZE, M. (2005) A variational discretization concept in control constrained optimization: the linear-quadratic case. Comput. Optim. Appl., 30, 45-61.

Hurri-Syrjänen, R. \& VÄHÄKangas, A. V. (2013) On fractional Poincaré inequalities. J. Anal. Math., 120, 85-104.

Jin, B., LAZAROv, R., PAScIAK, J. \& ZHOU, Z. (2015) Error analysis of semidiscrete finite element methods for inhomogeneous time-fractional diffusion. IMA J. Numer. Anal., 35, 561-582. 
Jin, B., LAZAROV, R. \& ZHOU, Z. (2016) Two fully discrete schemes for fractional diffusion and diffusion-wave equations with nonsmooth data. SIAM J. Sci. Comput., 38, A146-A170.

JIN, B., LI, B. \& ZHOU, Z. (2018a) Discrete maximal regularity of time-stepping schemes for fractional evolution equations. Numer. Math., 138, 101-131.

JIN, B., LI, B. \& ZHOU, Z. (2018b) Numerical analysis of nonlinear subdiffusion equations. SIAM J. Numer. Anal., 56, 1-23.

Kilbas, A. A., Srivastava, H. M. \& Trujillo, J. J. (2006) Theory and Applications of Fractional Differential Equations. Elsevier Science B.V., Amsterdam.

KovÁcs, B., Li, B. \& LuBICH, C. (2016) A-stable time discretizations preserve maximal parabolic regularity. SIAM J. Numer. Anal., 54, 3600-3624.

Kunstmann, P. C., LI, B. \& Lubich, C. (2018) Runge-Kutta time discretization of nonlinear parabolic equations studied via discrete maximal parabolic regularity. Found. Comput. Math., DOI: 10.1007/s10208-0179364-x.

Kunstmann, P. C. \& Weis, L. (2004) Maximal $L_{p}$-regularity for parabolic equations, Fourier multiplier theorems and $H^{\infty}$-functional calculus. Functional Analytic Methods for Evolution Equations. Lecture Notes in Math., vol. 1855. Springer, Berlin, pp. 65-311.

LiN, Y. \& XU, C. (2007) Finite difference/spectral approximations for the time-fractional diffusion equation. $J$. Comput. Phys., 225, 1533-1552.

LÜ, Q. \& ZuAZuA, E. (2016) On the lack of controllability of fractional in time ODE and PDE. Math. Control Signals Systems, 28, Art. 10, 21.

LUBICH, C. (1986) Discretized fractional calculus. SIAM J. Math. Anal., 17, 704-719.

MEIDNER, D. \& VEXler, B. (2008) A priori error estimates for space-time finite element discretization of parabolic optimal control problems. II. Problems with control constraints. SIAM J. Control Optim., 47, 1301-1329.

Nigmatulin, R. R. (1986) The realization of the generalized transfer equation in a medium with fractal geometry. Phys. Stat. Sol. B, 133, 425-430.

TARTAR, L. (2006) An Introduction to Navier-Stokes Equation and Oceanography. Springer-Verlag, Berlin.

WEIS, L. (2001) Operator-valued Fourier multiplier theorems and maximal $L_{p}$-regularity. Math. Ann., 319, $735-758$.

YE, X. \& XU, C. (2013) Spectral optimization methods for the time fractional diffusion inverse problem. Numer. Math. Theory Methods Appl., 6, 499-516.

YE, X. \& XU, C. (2015) A space-time spectral method for the time fractional diffusion optimal control problems. Adv. Difference Equ., 156.

ZHOU, Z. \& GoNG, W. (2016) Finite element approximation of optimal control problems governed by time fractional diffusion equation. Comput. Math. Appl., 71, 301-318.

ZIEMER, W. P. (1989) Weakly Differentiable Functions. Springer-Verlag, New York. 